\title{
Study of the Mass and Spin-Parity of the Higgs Boson Candidate via Its Decays to $Z$ Boson Pairs
}

\author{
S. Chatrchyan et al.* \\ (CMS Collaboration) \\ (Received 29 December 2012; published 21 February 2013)
}

\begin{abstract}
A study is presented of the mass and spin-parity of the new boson recently observed at the LHC at a mass near $125 \mathrm{GeV}$. An integrated luminosity of $17.3 \mathrm{fb}^{-1}$, collected by the CMS experiment in protonproton collisions at center-of-mass energies of 7 and $8 \mathrm{TeV}$, is used. The measured mass in the $Z Z$ channel, where both $Z$ bosons decay to $e$ or $\mu$ pairs, is $126.2 \pm 0.6$ (stat) \pm 0.2 (syst) GeV. The angular distributions of the lepton pairs in this channel are sensitive to the spin-parity of the boson. Under the assumption of spin 0 , the present data are consistent with the pure scalar hypothesis, while disfavoring the pure pseudoscalar hypothesis.
\end{abstract}

DOI: 10.1103/PhysRevLett.110.081803

PACS numbers: 14.80.Bn, 12.60.-i, 13.85.Qk, 13.88.+e

Recently the ATLAS and CMS Collaborations announced the observation of a narrow resonance with mass near $125 \mathrm{GeV}[1,2]$ and properties consistent with those of the Higgs boson predicted in the standard model (SM) [3-5] of particle physics. This observation may help to elucidate the nature of spontaneous electroweak symmetry breaking [6-11]. The main decay modes by which this resonance is observed include photon pairs $(\gamma \gamma)$ and massive vector boson pairs ( $W W$ and $Z Z$ ), where at least one of the vector bosons is off mass shell. As more proton-proton collision data are recorded at the Large Hadron Collider (LHC), attention is turning to the determination of various properties of this state, including its mass, spin, parity, and couplings to SM particles.

The observation of the new boson in the $\gamma \gamma$ channel implies that the resonance must be a boson with spin 0 or 2 ; spin 1 is excluded by the Landau-Yang theorem $[12,13]$. The decays of the new boson to $Z Z$ in which both $Z$ bosons decay to charged-lepton pairs $\left(\ell^{+} \ell^{-}\right.$, where $\ell=e$ or $\left.\mu\right)$ offer the possibility to probe the spin-parity and mass of the resonance. We describe these measurements in this Letter, using a data set recorded by the CMS experiment in proton-proton collisions at the LHC, corresponding to an integrated luminosity of $17.3 \mathrm{fb}^{-1}$, with $5.1 \mathrm{fb}^{-1}$ collected at a center-of-mass energy of $7 \mathrm{TeV}$ and $12.2 \mathrm{fb}^{-1}$ at $8 \mathrm{TeV}$.

The compact muon solenoid (CMS) detector, described in detail elsewhere [14], is a large general-purpose device based on a silicon pixel and strip tracking system, a lead tungstate crystal electromagnetic calorimeter (ECAL), and a brass and scintillator hadron calorimeter, all inside the field volume of a $3.8 \mathrm{~T}$ solenoidal magnet. Outside the

*Full author list given at the end of the article.

Published by the American Physical Society under the terms of the Creative Commons Attribution 3.0 License. Further distribution of this work must maintain attribution to the author(s) and the published article's title, journal citation, and DOI. magnet is a multilayered muon detection system embedded in steel absorber plates, which form the return path for the magnetic flux, as well as forward calorimetry. The detector is particularly well suited for measuring electron and muon transverse momenta $\left(p_{T}\right)$ over a wide range.

The signal candidates are selected using well-identified and isolated prompt leptons. The event selection and lepton reconstruction are described elsewhere [2]. Events are selected online by triggers requiring the presence of either an $e e, e \mu$, or $\mu \mu$ pair with asymmetric $p_{T}$ thresholds, or three electrons with reduced thresholds. The reconstructed electrons are required to have $p_{T}^{e}>7 \mathrm{GeV}$ and to be within the tracker geometrical acceptance, at pseudorapidities $\left|\eta^{e}\right|<2.5$, where $\eta \equiv-\ln [\tan (\theta / 2)]$ in terms of the polar angle $\theta$. The corresponding requirements for reconstructed muons are $p_{T}^{\mu}>5 \mathrm{GeV}$ and $\left|\eta^{\mu}\right|<2.4$. The selection requires the presence of two pairs of leptons. The leptons in a pair must be of opposite charge and same flavor. Photons with $p_{T}^{\gamma}>2 \mathrm{GeV}$ are reconstructed within $\left|\eta^{\gamma}\right|<$ 2.4 and considered as possible final-state radiation (FSR) candidates. An FSR photon is retained and associated with the closest lepton in a lepton pair only if the dilepton plus photon mass is closer to the nominal $Z$ boson mass. One lepton pair is required to be loosely consistent with originating from a $Z$ decay by demanding that the invariant mass of the pair be in the range $40-120 \mathrm{GeV}$. The first pair, denoted $Z_{1}$, is the one nearest the $Z$ in mass. The second pair, denoted $Z_{2}$, is required to satisfy $12<m_{Z_{2}}<$ $120 \mathrm{GeV}$. Among the four selected leptons forming the two $Z$ boson candidates, at least one should have $p_{T}>$ $20 \mathrm{GeV}$ and another should have $p_{T}>10 \mathrm{GeV}$.

The selected sample is dominated by continuum electroweak production of $Z Z / Z \gamma^{*}$, which constitutes irreducible background, estimated from Monte Carlo simulation as in the previous analysis [2]. A small background from reducible sources remains, mainly from $Z+X$ events, where $X$ consists of two reconstructed leptons, at least one of which is a nonprompt lepton, including misidentified leptons, leptons from heavy-quark decays, or photon 
conversions. The reducible background is measured from signal-free control regions in experimental data [2].

The performance of the signal selection and background suppression has been improved compared with the previous analysis [2] by using a three-electron trigger, using better muon reconstruction and momentum measurement algorithms, fine-tuning the electron isolation requirement, and by using a regression technique, as previously used for the $H \rightarrow \gamma \gamma$ analysis [2], for the contribution of the ECAL to the electron momentum measurement. For similar reducible background rates, the absolute signal detection efficiency is improved by up to $4 \%$ in the $4 e$ channel and up to $2 \%$ in the $2 e 2 \mu$ channel. The resolution of the reconstructed mass of the $4 \ell$ system is improved, relatively, by about $10 \%$ in the $4 e$ and $2 e 2 \mu$ channels. Signal candidate masses are measured with a per-event mass precision varying between $1 \%$ and $3 \%$. The detection efficiency for a SM Higgs boson of $m=126 \mathrm{GeV}$, with leptons within the geometrical acceptance, is $31 \%$ in the $4 e$ channel, $42 \%$ in the $2 e 2 \mu$ channel, and $59 \%$ in the $4 \mu$ channel.

Systematic uncertainties are evaluated from the observed data for the trigger efficiency $(1.5 \%)$ and the combined lepton reconstruction, identification, and isolation efficiencies. These range from $1.2 \%$ in the $4 \mu$ channel to about $11 \%$ in the $4 e$ channel. Systematic uncertainties on energy-momentum calibration and energy resolution are incorporated through their effects on the reconstructed mass distributions. Uncertainties of $0.2 \%, 0.2 \%$, and $0.1 \%$, are assigned on the mass scale for the $4 e, 2 e 2 \mu$, and $4 \mu$ channels, respectively. The effect of the energy resolution uncertainties is taken into account by incorporating a $20 \%$ uncertainty on the simulated width of the signal mass peak. To validate the level of accuracy with which the absolute mass scale and resolution are known [2,15], we use $Z \rightarrow \ell \ell, Y \rightarrow \ell \ell$, and $J / \psi \rightarrow \ell \ell$ events. The limited statistical precision of the control samples is included as a systematic uncertainty on the final results. Since the reducible background is derived from control regions, its prediction is independent of the uncertainties on the integrated luminosity. The integrated luminosity uncertainty $(2.2 \%$ at $7 \mathrm{TeV}$ [16] and $4.4 \%$ at $8 \mathrm{TeV}$ [17]) enters the evaluation of the expected $Z Z$ background and signal rates. Systematic uncertainties on the Higgs boson cross section (about 18\%) and branching fraction (2\%) are taken from Refs. [18,19].

Figure 1(a) shows the invariant mass distribution of the selected four-lepton events in the mass range $70<m_{4 \ell}<$ $180 \mathrm{GeV}$. The contribution expected from a SM Higgs boson of mass $m=126 \mathrm{GeV}$ is displayed. The peak from $Z \rightarrow 4 \ell$ decay, studied in detail elsewhere [20], is observed at the nominal $Z$ boson mass. The signal from the new boson is a distinct peak above the expected background, consistent with the signal line shape depicted in the figure. The background is locally flat and dominated by
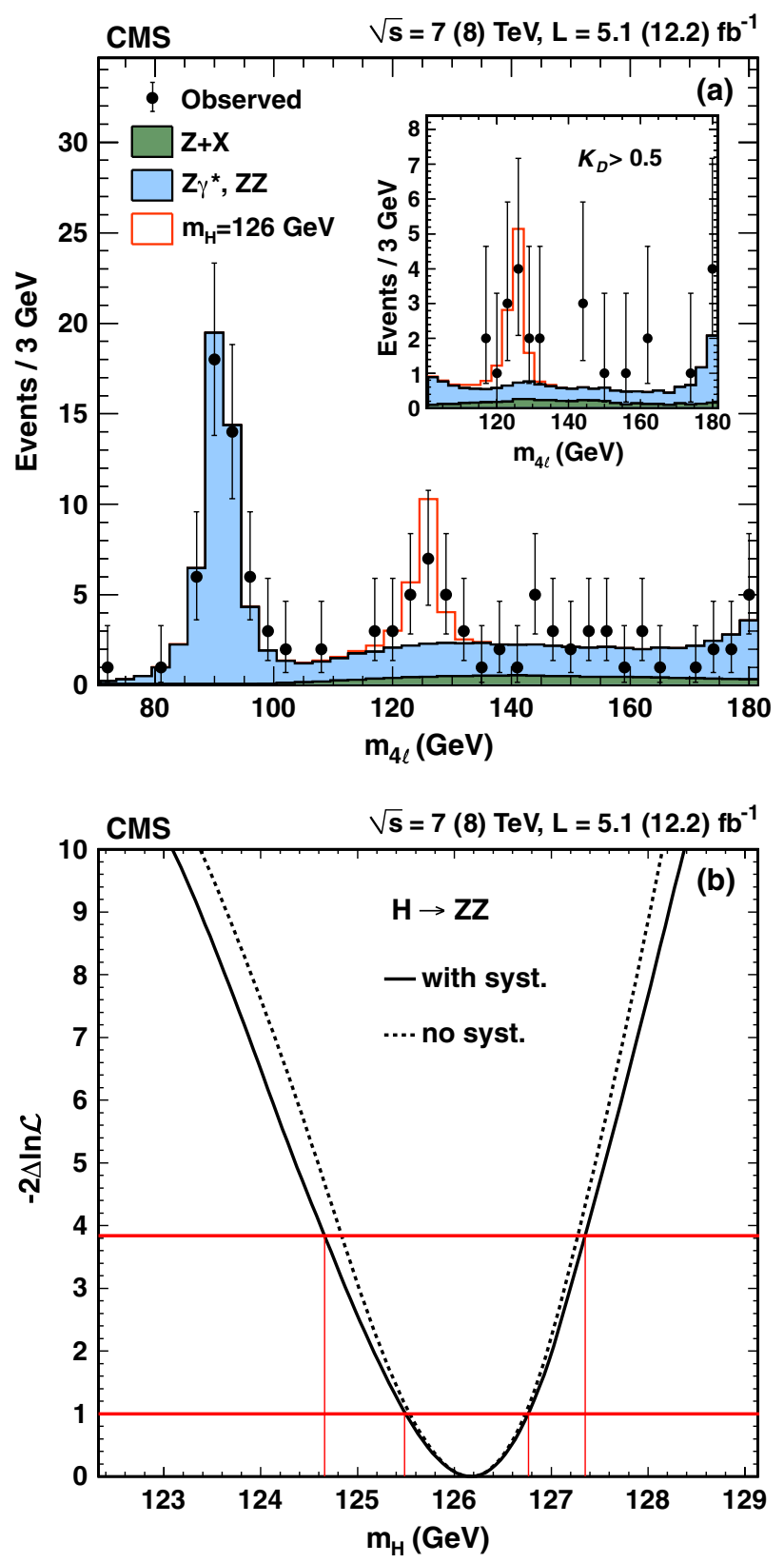

FIG. 1 (color online). (a) Distribution of four-lepton invariant mass in the range near the $126 \mathrm{GeV}$ resonance. Points represent the observed data, shaded histograms represent the backgrounds, and the open histograms represent the signal expectation. The inset shows the $m_{4 \ell}$ distribution for events with high values of kinematic discriminant $K_{D}$. (b) Scan of $-2 \Delta \ln \mathcal{L}$ versus $m_{H}$ with and without the effect of systematic uncertainties included.

the $Z Z / Z \gamma^{*}$ contribution. In the mass range, $121.5<$ $m_{4 \ell}<130.5 \mathrm{GeV}$, corresponding to the three central bins around the new boson peak in Fig. 1(a), we observe 17 events: there are 6,8 , and 3 events in the $4 \mu, 2 e 2 \mu$, and $4 e$ final states, respectively. This compares to an expectation of $6.8 \pm 0.8$ (stat) \pm 0.3 (syst) from SM background.

Further separation between the signal and background is provided by a discriminant $K_{D}$ that incorporates the 
production and decay kinematics. In this analysis, we make use of observables defined for each event in the $4 \ell$ centerof-mass frame; the rapidity and transverse momentum of the $4 \ell$ system depend on the production mechanism and are ignored. We use a matrix element likelihood approach [2,21-23], which combines, for each value of $m_{4 \ell}$, the two dilepton masses $m_{Z_{1}}$ and $m_{Z_{2}}$ and five angular variables denoted $\vec{\Omega}$. We introduce a kinematic discriminant $K_{D}$ using the probability density in the dilepton masses and angular variables, $\mathcal{P}\left(m_{Z_{1}}, m_{Z_{2}}, \vec{\Omega} \mid m_{4 \ell}\right)$. The discriminant is defined as

$K_{D} \equiv \frac{\mathcal{P}_{\text {sig }}}{\mathcal{P}_{\text {sig }}+\mathcal{P}_{\text {bkg }}}=\left[1+\frac{\mathcal{P}_{\mathrm{bkg}}\left(m_{Z_{1}}, m_{Z_{2}}, \vec{\Omega} \mid m_{4 \ell}\right)}{\mathcal{P}_{\text {sig }}\left(m_{Z_{1}}, m_{Z_{2}}, \vec{\Omega} \mid m_{4 \ell}\right)}\right]^{-1}$.

A scalar SM Higgs boson is assumed for the signal. The separation between the signal and background is relatively insensitive to the particular choice of a signal spin-parity hypothesis [22]. The minimum $p$ value [24], which characterizes the probability for a background fluctuation to be at least as large as the observed maximum excess around $m \simeq 126 \mathrm{GeV}$, is obtained from the measurements of $m_{4 \ell}$ and $K_{D}$. It corresponds to a significance of 4.5 standard deviations, which is to be compared to an expected significance of 5.0 standard deviations for the SM Higgs boson.

We measure the mass of the boson using a maximumlikelihood fit to three-dimensional distributions combining for each event the $m_{4 \ell}$, the associated per-event uncertainties $\delta m_{4 \ell}$ [15] calculated from the individual lepton momentum errors, and $K_{D}$. The signal strength $\mu$ (defined below) is a free parameter in this mass fit. A scalar SM Higgs boson is assumed for the signal line shape. Figure 1(b) shows the value of $-2 \Delta \ln \mathcal{L}$, where $\mathcal{L}$ is the likelihood, as a function of $m_{H}$, with and without the effects of systematic uncertainties included. An estimate for the mass of $126.2 \pm 0.6$ (stat) \pm 0.2 (syst) $\mathrm{GeV}$ is obtained.
Combined with the result from the $\gamma \gamma$ channel [2], we obtain a mass of $125.8 \pm 0.4$ (stat) \pm 0.4 (syst) GeV. This value improves upon and supersedes the previous result.

We then compare the observations with the expectation for the SM Higgs boson at the mass value fixed to $125.8 \mathrm{GeV}$, and obtain a measurement of the signal strength $\mu=\sigma / \sigma_{\mathrm{SM}}$, the production cross section times the branching fraction relative to the SM expectation. This is evaluated from a scan of a profile likelihood ratio. We perform an unbinned maximum-likelihood fit of the twodimensional distributions $\mathcal{P}\left(m_{4 \ell} \mid m_{H}\right) \times \mathcal{P}\left(K_{D} \mid m_{4 \ell}\right)$ for the signal, and $\mathcal{P}\left(m_{4 \ell}\right) \times \mathcal{P}\left(K_{D} \mid m_{4 \ell}\right)$ for the background. The fit is performed simultaneously in the $4 e, 2 e 2 \mu$, and $4 \mu$ channels. We obtain a signal strength of $\mu=0.80_{-0.28}^{+0.35}$, consistent with the expectation for a SM Higgs boson.

The kinematics of the production and decay of the new boson in the $Z Z \rightarrow 4 \ell$ channel are sensitive to its spin and parity [21-23,25-35]. To distinguish any two spin-parity hypotheses, we use discriminants of the form $\mathcal{D}_{12}=\mathcal{P}_{1} /\left(\mathcal{P}_{1}+\mathcal{P}_{2}\right)$, where $\mathcal{P}_{1}$ and $\mathcal{P}_{2}$ are the probability densities in $m_{Z_{1}}, m_{Z_{2}}$, and $\vec{\Omega}$ corresponding to the two spin-parity hypotheses we wish to discriminate and include parametrizations of the $m_{4 \ell}$ distribution for a resonance at the mass of the new boson. We define two spinparity discriminants: $D_{\mathrm{PS}}$ for the discrimination between a SM Higgs boson and a pure pseudoscalar state $J^{P}=0^{-}$; $D_{\mathrm{GS}}$ for discrimination between a SM Higgs boson and a spin-two tensor state $J^{P}=2^{+}$with the minimal gravitonlike coupling to gluons in production and to $Z$ bosons in decay. We also define a discriminant $D_{\mathrm{SB}}=\mathcal{P}_{\text {sig }} /\left(\mathcal{P}_{\text {sig }}+\right.$ $\mathcal{P}_{\text {bkg }}$ ), similar to $K_{D}$ but where the probability densities also include $m_{4 \ell}$, for the discrimination between a SM Higgs boson, with $J^{P}=0^{+}$, and the background.

We then fit the observed data in a two-dimensional plane of $D_{\mathrm{PS}}$ or $D_{\mathrm{GS}}$ versus $D_{\mathrm{SB}}$ in the mass range $106<m_{4 \ell}<$ $141 \mathrm{GeV}$ and obtain the likelihood values $\mathcal{L}_{1}$ and $\mathcal{L}_{2}$ for
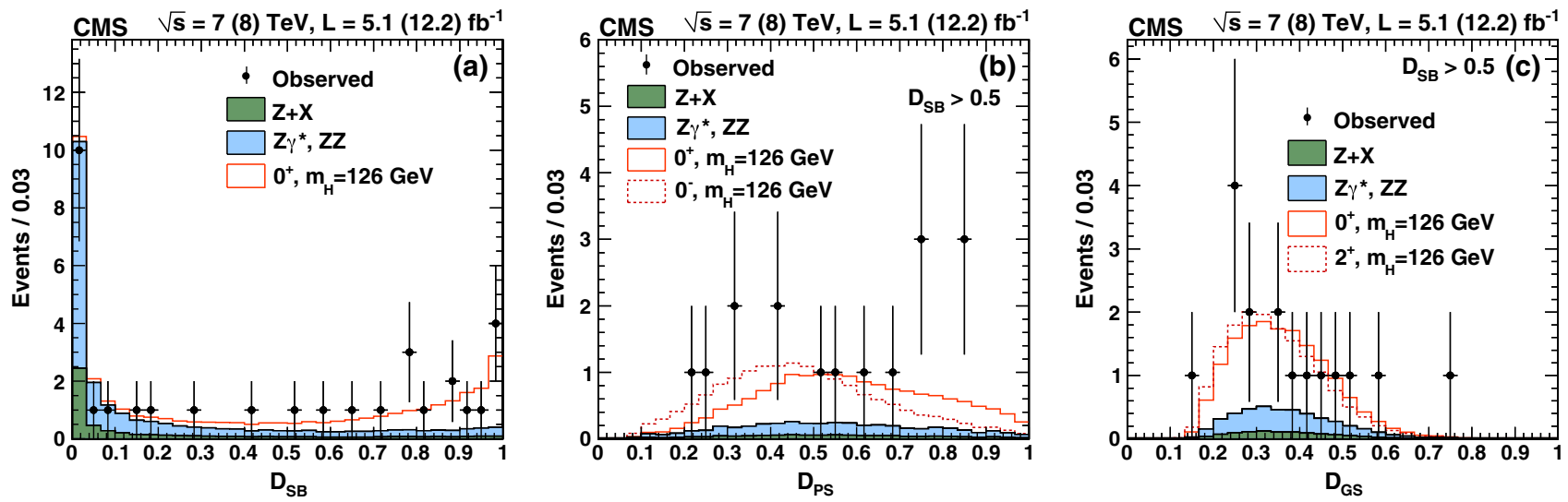

FIG. 2 (color online). (a) Observed distribution of the $D_{\mathrm{SB}}$ (SM Higgs boson versus background) discriminant compared with the background and signal expectations. (b) Observed distribution of $D_{\mathrm{PS}}\left(J^{P}=0^{-}\right.$versus $\left.J^{P}=0^{+}\right)$compared with expectation, for $D_{\mathrm{SB}}>0.5$. (c) Observed distribution of $D_{\mathrm{GS}}\left(J^{P}=2^{+}\right.$versus $\left.J^{P}=0^{+}\right)$compared with expectation, for $D_{\mathrm{SB}}>0.5$. Points represent the observed data, shaded histograms represent the background, and the open histograms represent the expectation for a $126 \mathrm{GeV}$ boson with the indicated spin-parity, produced at the SM Higgs boson rate. 


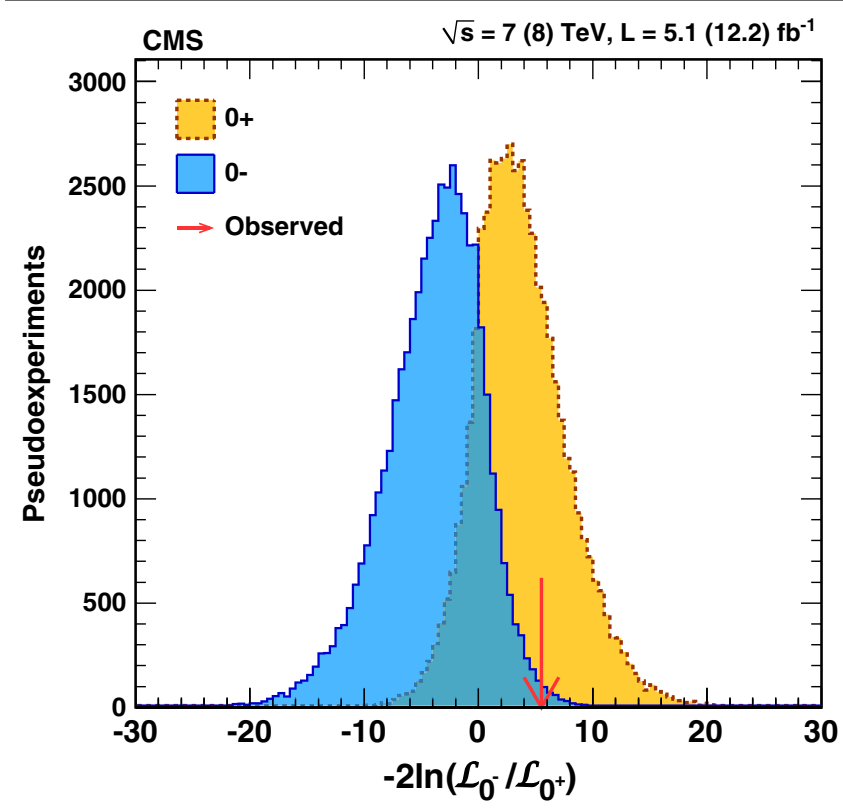

FIG. 3 (color online). Expected distribution of $-2 \ln \mathcal{L}_{0^{-}} / \mathcal{L}_{0^{+}}$ under the pure pseudoscalar and pure scalar hypotheses (histograms). The arrow indicates the value determined from the observed data.

two hypotheses of each signal type plus background. Figure 2(a) shows the observed projections of $D_{\mathrm{SB}}$ for events in this mass range, and for a SM Higgs boson signal with $m=126 \mathrm{GeV}$. Figures 2(b) and 2(c) show the projections of the $D_{\mathrm{PS}}$ and $D_{\mathrm{GS}}$ discriminants, for events with $D_{\mathrm{SB}}>0.5$. In these latter two cases, the distributions for the spin-parity states being distinguished are also illustrated in the plot. More data are needed for significant discrimination of the $0^{+}$from the $2^{+}$hypothesis.

Figure 3 shows the distributions of the log-likelihood ratio $-2 \ln \mathcal{L}_{0^{-}} / \mathcal{L}_{0^{+}}$from pseudoexperiments under the assumptions of either a pure scalar or a pure pseudoscalar model. The arrow indicates the observed value. Under the assumption of spin 0 , the test statistic formed from a profile likelihood ratio $\lambda=\mathcal{L}_{0^{-}} / \mathcal{L}_{0^{+}}$of the $0^{-}$and $0^{+}$hypotheses yields a $p$ value of $0.072 \%$ for $0^{-}$and a $p$ value of 0.7 for $0^{+}$, with $-2 \ln \lambda=5.5$ favoring $0^{+}$. This corresponds to a $\mathrm{CL}_{s}$ [36] value of $2.4 \%$, a more conservative value for judging whether the observed data are compatible with $0^{-}$. The results presented here have been confirmed with independent methods [37] based on leading-order matrix elements [38].

In summary, we have measured the mass of the new boson to be $126.2 \pm 0.6$ (stat) \pm 0.2 (syst) $\mathrm{GeV}$ in the $Z Z$ channel, where both $Z$ bosons decay to lepton pairs. Combining results from the $\gamma \gamma$ and $Z Z$ channels, we obtain a mass of $125.8 \pm 0.4$ (stat) \pm 0.4 (syst) $\mathrm{GeV}$, which improves upon previously published results. At this mass the signal strength $\mu=\sigma / \sigma_{\mathrm{SM}}$ is measured to be $\mu=0.80_{-0.28}^{+0.35}$. Under the assumption of spin zero, the observed data are consistent with the pure scalar hypothesis, while disfavoring the pure pseudoscalar hypothesis. This is the first study of the spinparity of the newly discovered boson.

We congratulate our colleagues in the CERN accelerator departments for the excellent performance of the LHC and thank the technical and administrative staffs at CERN and at other CMS institutes for their contributions to the success of the CMS effort. In addition, we gratefully acknowledge the computing centers and personnel of the Worldwide LHC Computing Grid for delivering so effectively the computing infrastructure essential to our analyses. Finally, we acknowledge the enduring support for the construction and operation of the LHC and the CMS detector provided by the following funding agencies: BMWF and FWF (Austria); FNRS and FWO (Belgium); CNPq, CAPES, FAPERJ, and FAPESP (Brazil); MEYS (Bulgaria); CERN; CAS, MoST, and NSFC (China); COLCIENCIAS (Colombia); MSES (Croatia); RPF (Cyprus); MoER, SF0690030s09 and ERDF (Estonia); Academy of Finland, MEC, and HIP (Finland); CEA and CNRS/IN2P3 (France); BMBF, DFG, and HGF (Germany); GSRT (Greece); OTKA and NKTH (Hungary); DAE and DST (India); IPM (Iran); SFI (Ireland); INFN (Italy); NRF and WCU (Korea); LAS (Lithuania); CINVESTAV, CONACYT, SEP, and UASLP-FAI (Mexico); MSI (New Zealand); PAEC (Pakistan); MSHE and NSC (Poland); FCT (Portugal); JINR (Armenia, Belarus, Georgia, Ukraine, Uzbekistan); MON, RosAtom, RAS and RFBR (Russia); MSTD (Serbia); SEIDI and CPAN (Spain); Swiss Funding Agencies (Switzerland); NSC (Taipei); ThEP, IPST and NECTEC (Thailand); TUBITAK and TAEK (Turkey); NASU (Ukraine); STFC (United Kingdom); DOE and NSF (U.S.).

[1] ATLAS Collaboration, Phys. Lett. B 716, 1 (2012).

[2] CMS Collaboration, Phys. Lett. B 716, 30 (2012).

[3] S. L. Glashow, Nucl. Phys. 22, 579 (1961).

[4] S. Weinberg, Phys. Rev. Lett. 19, 1264 (1967).

[5] A. Salam, in Proceedings of the Eighth Nobel Symposium: Elementary Particle Physics: Relativistic Groups and Analyticity, edited by N. Svartholm (Almqvist \& Wiskell, Stockholm, 1968), p. 367.

[6] P. W. Higgs, Phys. Lett. 12, 132 (1964).

[7] P. W. Higgs, Phys. Rev. Lett. 13, 508 (1964).

[8] P. W. Higgs, Phys. Rev. 145, 1156 (1966).

[9] F. Englert and R. Brout, Phys. Rev. Lett. 13, 321 (1964).

[10] G. S. Guralnik, C. R. Hagen, and T. W. B. Kibble, Phys. Rev. Lett. 13, 585 (1964).

[11] T. W. B. Kibble, Phys. Rev. 155, 1554 (1967).

[12] L. D. Landau, Dokl. Akad. Nauk SSSR 60, 207 (1948).

[13] C.-N. Yang, Phys. Rev. 77, 242 (1950).

[14] CMS Collaboration, JINST 3, S08004 (2008).

[15] CMS Collaboration, Phys. Rev. Lett. 108, 111804 (2012).

[16] CMS Collaboration, CMS Physics Analysis Summary Report No. CMS-PAS-SMP-12-008, 2012 [http://cdsweb .cern.ch/record/1434360]. 
[17] CMS Collaboration, CMS Physics Analysis Summary Report No. CMS-PAS-LUM-12-001, 2012 [http:// cdsweb.cern.ch/record/1482193].

[18] LHC Higgs Cross Section Working Group, CERN Report No. CERN-2011-002, 2011 [http://cdsweb.cern.ch/ record/1318996].

[19] A. Denner, S. Heinemeyer, I. Puljak, D. Rebuzzi, and M. Spira, Eur. Phys. J. C 71, 1753 (2011).

[20] CMS Collaboration, J. High Energy Phys. 12 (2012) 034.

[21] Y. Gao, A. V. Gritsan, Z. Guo, K. Melnikov, M. Schulze, and N. V. Tran, Phys. Rev. D 81, 075022 (2010).

[22] S. Bolognesi, Y. Gao, A. V. Gritsan, K. Melnikov, M. Schulze, N. V. Tran, and A. Whitbeck, Phys. Rev. D 86, 095031 (2012).

[23] Y. Chen, N. Tran, and R. Vega-Morales, arXiv:1211.1959.

[24] J. Beringer et al. (Particle Data Group), Phys. Rev. D 86, 010001 (2012).

[25] A. Soni and R. M. Xu, Phys. Rev. D 48, 5259 (1993).

[26] V.D. Barger, K. Cheung, A. Djouadi, B. A. Kniehl, and P. M. Zerwas, Phys. Rev. D 49, 79 (1994).

[27] S. Y. Choi, D. J. Miller, M. M. Muhlleitner, and P. M. Zerwas, Phys. Lett. B 553, 61 (2003).
[28] B. C. Allanach, K. Odagiri, M. J. Palmer, M. A. Parker, A. Sabetfakhri, and B.R. Webber, J. High Energy Phys. 12 (2002) 039.

[29] C. P. Buszello, I. Fleck, P. Marquard, and J. J. van der Bij, Eur. Phys. J. C 32, 209 (2004).

[30] R. M. Godbole, D. J. Miller, and M. M. Muhlleitner, J. High Energy Phys. 12 (2007) 031.

[31] W.-Y. Keung, I. Low, and J. Shu, Phys. Rev. Lett. 101, 091802 (2008).

[32] O. Antipin and A. Soni, J. High Energy Phys. 10 (2008) 018.

[33] K. Hagiwara, Q. Li, and K. Mawatari, J. High Energy Phys. 07 (2009) 101.

[34] A. De Rujula, J. Lykken, M. Pierini, C. Rogan, and M. Spiropulu, Phys. Rev. D 82, 013003 (2010).

[35] J.S. Gainer, K. Kumar, I. Low, and R. Vega-Morales, J. High Energy Phys. 11 (2011) 027.

[36] A. L. Read, J. Phys. G 28, 2693 (2002).

[37] P. Avery, D. Bourilkov, M. Chen, T. Cheng, A. Drozdetskiy, J. S. Gainer, A. Korytov, K. T. Matchev, P. Milenovic, G. Mitselmakher, M. Park, and A. Rinkevicius, arXiv:1210.0896.

[38] J. Alwall, P. Demin, S. de Visscher, R. Frederix, M. Herquet, F. Maltoni, T. Plehn, D. L. Rainwater, and T. Stelzer, J. High Energy Phys. 09 (2007) 028.

S. Chatrchyan, ${ }^{1}$ V. Khachatryan, ${ }^{1}$ A. M. Sirunyan, ${ }^{1}$ A. Tumasyan,,${ }^{1}$ W. Adam,${ }^{2}$ E. Aguilo, ${ }^{2}$ T. Bergauer, ${ }^{2}$ M. Dragicevic, ${ }^{2}$ J. Erö, ${ }^{2}$ C. Fabjan, ${ }^{2, b}$ M. Friedl, ${ }^{2}$ R. Frühwirth, ${ }^{2, b}$ V. M. Ghete, ${ }^{2}$ N. Hörmann, ${ }^{2}$ J. Hrubec, ${ }^{2}$ M. Jeitler, ${ }^{2, b}$ W. Kiesenhofer, ${ }^{2}$ V. Knünz, ${ }^{2}$ M. Krammer, ${ }^{2, b}$ I. Krätschmer, ${ }^{2}$ D. Liko, ${ }^{2}$ I. Mikulec, ${ }^{2}$ M. Pernicka, ${ }^{2, a}$ D. Rabady, ${ }^{2, \mathrm{c}}$ B. Rahbaran, ${ }^{2}$ C. Rohringer, ${ }^{2}$ H. Rohringer, ${ }^{2}$ R. Schöfbeck, ${ }^{2}$ J. Strauss, ${ }^{2}$ A. Taurok, ${ }^{2}$ W. Waltenberger, ${ }^{2}$ C.-E. Wulz, ${ }^{2, b}$ V. Mossolov, ${ }^{3}$ N. Shumeiko, ${ }^{3}$ J. Suarez Gonzalez, ${ }^{3}$ S. Alderweireldt, ${ }_{4}^{4}$ M. Bansal, ${ }^{4}$ S. Bansal, ${ }^{4}$

T. Cornelis, ${ }^{4}$ E. A. De Wolf, ${ }^{4}$ X. Janssen, ${ }^{4}$ S. Luyckx, ${ }^{4}$ L. Mucibello, ${ }^{4}$ S. Ochesanu, ${ }^{4}$ B. Roland, ${ }^{4}$ R. Rougny, ${ }^{4}$

H. Van Haevermaet, ${ }^{4}$ P. Van Mechelen, ${ }^{4}$ N. Van Remortel, ${ }^{4}$ A. Van Spilbeeck, ${ }^{4}$ F. Blekman, ${ }_{5}^{5}$ S. Blyweert, ${ }^{5}$

J. D'Hondt, ${ }^{5}$ R. Gonzalez Suarez, ${ }^{5}$ A. Kalogeropoulos, ${ }^{5}$ M. Maes, ${ }^{5}$ A. Olbrechts, ${ }^{5}$ S. Tavernier, ${ }^{5}$ W. Van Doninck, ${ }^{5}$ P. Van Mulders, ${ }^{5}$ G. P. Van Onsem, ${ }^{5}$ I. Villella, ${ }^{5}$ B. Clerbaux, ${ }^{6}$ G. De Lentdecker, ${ }^{6}$ V. Dero, ${ }_{6}^{6}$ A. P. R. Gay, ${ }^{6}$ T. Hreus, ${ }^{6}$

A. Léonard, ${ }^{6}$ P. E. Marage, ${ }^{6}$ A. Mohammadi, ${ }^{6}$ T. Reis, ${ }^{6}$ L. Thomas, ${ }^{6}$ C. Vander Velde,${ }^{6}$ P. Vanlaer, ${ }^{6}$ J. Wang, ${ }^{6}$

V. Adler ${ }^{7}$ K. Beernaert, ${ }^{7}$ A. Cimmino, ${ }^{7}$ S. Costantini, ${ }^{7}$ G. Garcia, ${ }^{7}$ M. Grunewald, ${ }^{7}$ B. Klein, ${ }^{7}$ J. Lellouch, ${ }^{7}$ A. Marinov, ${ }^{7}$ J. Mccartin, ${ }^{7}$ A. A. Ocampo Rios, ${ }^{7}$ D. Ryckbosch, ${ }^{7}$ M. Sigamani, ${ }^{7}$ N. Strobbe, ${ }^{7}$ F. Thyssen, ${ }^{7}$ M. Tytgat, ${ }^{7}$ S. Walsh, ${ }^{7}$ E. Yazgan, ${ }^{7}$ N. Zaganidis,${ }^{7}$ S. Basegmez, ${ }^{8}$ G. Bruno, ${ }^{8}$ R. Castello, ${ }^{8}$ L. Ceard, ${ }^{8}$ C. Delaere, ${ }^{8}$ T. du Pree,${ }^{8}$

D. Favart, ${ }^{8}$ L. Forthomme, ${ }^{8}$ A. Giammanco, ${ }^{8, d}$ J. Hollar, ${ }^{8}$ V. Lemaitre, ${ }^{8}$ J. Liao, ${ }^{8}$ O. Militaru, ${ }^{8}$ C. Nuttens, ${ }^{8}$

D. Pagano, ${ }^{8}$ A. Pin,${ }^{8}$ K. Piotrzkowski, ${ }^{8}$ M. Selvaggi,${ }^{8}$ J. M. Vizan Garcia, ${ }^{8}$ N. Beliy, ${ }^{9}$ T. Caebergs, ${ }^{9}$ E. Daubie, ${ }^{9}$ G. H. Hammad, ${ }^{9}$ G. A. Alves, ${ }^{10}$ M. Correa Martins Junior, ${ }^{10}$ T. Martins,${ }^{10}$ M. E. Pol, ${ }^{10}$ M. H. G. Souza,${ }^{10}$ W. L. Aldá Júnior, ${ }^{11}$ W. Carvalho, ${ }^{11}$ J. Chinellato,,${ }^{1, e}$ A. Custódio, ${ }^{11}$ E. M. Da Costa, ${ }^{11}$ D. De Jesus Damiao, ${ }^{11}$

C. De Oliveira Martins, ${ }^{11}$ S. Fonseca De Souza, ${ }^{11}$ H. Malbouisson, ${ }^{11}$ M. Malek, ${ }^{11}$ D. Matos Figueiredo, ${ }^{11}$ L. Mundim,${ }^{11}$ H. Nogima,${ }^{11}$ W. L. Prado Da Silva, ${ }^{11}$ A. Santoro,,${ }^{11}$ L. Soares Jorge,${ }^{11}$ A. Sznajder,${ }^{11}$

E. J. Tonelli Manganote, ${ }^{11, e}$ A. Vilela Pereira, ${ }^{11}$ T. S. Anjos, ${ }^{12 b}$ C. A. Bernardes, ${ }^{12 b}$ F. A. Dias, ${ }^{12 a, f}$

T. R. Fernandez Perez Tomei, ${ }^{12 a}$ E. M. Gregores, ${ }^{12 b}$ C. Lagana, ${ }^{12 a}$ F. Marinho, ${ }^{12 a}$ P. G. Mercadante, ${ }^{12 b}$ S. F. Novaes, ${ }^{12 a}$ Sandra S. Padula, ${ }^{12 a}$ V. Genchev,${ }^{13, c}$ P. Iaydjiev, ${ }^{13, c}$ S. Piperov,${ }^{13}$ M. Rodozov,${ }^{13}$ S. Stoykova, ${ }^{13}$

G. Sultanov, ${ }^{13}$ V. Tcholakov, ${ }^{13}$ R. Trayanov, ${ }^{13}$ M. Vutova, ${ }^{13}$ A. Dimitrov, ${ }^{14}$ R. Hadjiiska, ${ }^{14}$ V. Kozhuharov, ${ }^{14}$ L. Litov, ${ }^{14}$ B. Pavlov, ${ }^{14}$ P. Petkov, ${ }^{14}$ J. G. Bian, ${ }^{15}$ G. M. Chen, ${ }^{15}$ H. S. Chen, ${ }^{15}$ C. H. Jiang, ${ }^{15}$ D. Liang, ${ }^{15}$ S. Liang, ${ }^{15}$ X. Meng, ${ }^{15}$ J. Tao, ${ }^{15}$ J. Wang, ${ }^{15}$ X. Wang, ${ }^{15}$ Z. Wang, ${ }^{15}$ H. Xiao, ${ }^{15}$ M. Xu, ${ }^{15}$ J. Zang, ${ }^{15}$ Z. Zhang, ${ }^{15}$

C. Asawatangtrakuldee, ${ }^{16}$ Y. Ban, ${ }^{16}$ Y. Guo, ${ }^{16}$ W. Li,${ }^{16}$ S. Liu,${ }^{16}$ Y. Mao, ${ }^{16}$ S. J. Qian, ${ }^{16}$ H. Teng, ${ }^{16}$ D. Wang,,${ }^{16}$ L. Zhang, ${ }^{16}$ W. Zou, ${ }^{16}$ C. Avila, ${ }^{17}$ C. A. Carrillo Montoya, ${ }^{17}$ J. P. Gomez, ${ }^{17}$ B. Gomez Moreno, ${ }^{17}$

A. F. Osorio Oliveros,${ }^{17}$ J. C. Sanabria,${ }^{17}$ N. Godinovic,${ }^{18}$ D. Lelas,${ }^{18}$ R. Plestina, ${ }^{18, g}$ D. Polic, ${ }^{18}$ I. Puljak,${ }^{18}$ Z. Antunovic, ${ }^{19}$ M. Kovac, ${ }^{19}$ V. Brigljevic, ${ }^{20}$ S. Duric, ${ }^{20}$ K. Kadija, ${ }^{20}$ J. Luetic, ${ }^{20}$ D. Mekterovic, ${ }^{20}$ S. Morovic, ${ }^{20}$ 
L. Tikvica, ${ }^{20}$ A. Attikis, ${ }^{21}$ G. Mavromanolakis,${ }^{21}$ J. Mousa, ${ }^{21}$ C. Nicolaou, ${ }^{21}$ F. Ptochos,${ }^{21}$ P. A. Razis,${ }^{21}$ M. Finger,${ }^{22}$ M. Finger, Jr., ${ }^{22}$ Y. Assran, ${ }^{23, \mathrm{~h}}$ S. Elgammal, ${ }^{23, \mathrm{i}}$ A. Ellithi Kamel, ${ }^{23, \mathrm{j}}$ A. M. Kuotb Awad, ${ }^{23, \mathrm{k}}$ M. A. Mahmoud, ${ }^{23, \mathrm{k}}$ A. Radi,${ }^{23,1, \mathrm{~m}}$ M. Kadastik,${ }^{24}$ M. Müntel,${ }^{24}$ M. Murumaa, ${ }^{24}$ M. Raidal ${ }^{24}$ L. Rebane, ${ }^{24}$ A. Tiko, ${ }^{24}$ P. Eerola, ${ }^{25}$ G. Fedi, ${ }^{25}$ M. Voutilainen, ${ }^{25}$ J. Härkönen, ${ }^{26}$ A. Heikkinen, ${ }^{26}$ V. Karimäki, ${ }^{26}$ R. Kinnunen, ${ }^{26}$ M. J. Kortelainen, ${ }^{26}$ T. Lampén, ${ }^{26}$ K. Lassila-Perini, ${ }^{26}$ S. Lehti, ${ }^{26}$ T. Lindén, ${ }^{26}$ P. Luukka, ${ }^{26}$ T. Mäenpää, ${ }^{26}$ T. Peltola ${ }^{26}$ E. Tuominen, ${ }^{26}$ J. Tuominiemi, ${ }^{26}$ E. Tuovinen, ${ }^{26}$ D. Ungaro, ${ }^{26}$ L. Wendland, ${ }^{26}$ A. Korpela, ${ }^{27}$ T. Tuuva, ${ }^{27}$ M. Besancon, ${ }^{28}$ S. Choudhury, ${ }^{28}$ F. Couderc, ${ }^{28}$ M. Dejardin, ${ }^{28}$ D. Denegri, ${ }^{28}$ B. Fabbro, ${ }^{28}$ J. L. Faure, ${ }^{28}$ F. Ferri, ${ }^{28}$ S. Ganjour, ${ }^{28}$ A. Givernaud, ${ }^{28}$ P. Gras, ${ }^{28}$ G. Hamel de Monchenault, ${ }^{28}$ P. Jarry, ${ }^{28}$ E. Locci ${ }^{28}$ J. Malcles, ${ }^{28}$ L. Millischer, ${ }^{28}$ A. Nayak ${ }^{28}$ J. Rander, ${ }^{28}$ A. Rosowsky, ${ }^{28}$ M. Titov ${ }^{28}$ S. Baffioni, ${ }^{29}$ F. Beaudette, ${ }^{29}$ L. Benhabib,${ }^{29}$ L. Bianchini ${ }^{29}$ M. Bluj, ${ }^{29, n}$ P. Busson, ${ }^{29}$ C. Charlot,${ }^{29}$ N. Daci, ${ }^{29}$ T. Dahms, ${ }^{29}$ M. Dalchenko, ${ }^{29}$ L. Dobrzynski, ${ }^{29}$ A. Florent, ${ }^{29}$ R. Granier de Cassagnac, ${ }^{29}$ M. Haguenauer, ${ }^{29}$ P. Miné, ${ }^{29}$ C. Mironov, ${ }^{29}$ I. N. Naranjo, ${ }^{29}$ M. Nguyen,${ }^{29}$ C. Ochando, ${ }^{29}$ P. Paganini, ${ }^{29}$ D. Sabes,${ }^{29}$ R. Salerno, ${ }^{29}$ Y. Sirois, ${ }^{29}$ C. Veelken,${ }^{29}$ A. Zabi, ${ }^{29}$ J.-L. Agram,${ }^{30, o}$ J. Andrea, ${ }^{30}$ D. Bloch,${ }^{30}$ D. Bodin, ${ }^{30}$ J.-M. Brom, ${ }^{30}$ E. C. Chabert, ${ }^{30}$ C. Collard ${ }^{30}$ E. Conte, ${ }^{30, o}$ F. Drouhin, ${ }^{30, o}$ J.-C. Fontaine, ${ }^{30, o}$ D. Gelé, ${ }^{30}$ U. Goerlach, ${ }^{30}$ P. Juillot, ${ }^{30}$ A.-C. Le Bihan, ${ }^{30}$ P. Van Hove, ${ }^{30}$ S. Beauceron, ${ }^{31}$ N. Beaupere, ${ }^{31}$ O. Bondu, ${ }^{31}$ G. Boudoul,${ }^{31}$ S. Brochet, ${ }^{31}$ J. Chasserat,${ }^{31}$ R. Chierici, ${ }^{31, c}$ D. Contardo, ${ }^{31}$ P. Depasse,${ }^{31}$ H. El Mamouni, ${ }^{31}$ J. Fay,${ }^{31}$ S. Gascon, ${ }^{31}$ M. Gouzevitch, ${ }^{31}$ B. Ille, ${ }^{31}$ T. Kurca,${ }^{31}$ M. Lethuillier,${ }^{31}$ L. Mirabito, ${ }^{31}$ S. Perries, ${ }^{31}$ L. Sgandurra, ${ }^{31}$ V. Sordini, ${ }^{31}$ Y. Tschudi, ${ }^{31}$ P. Verdier, ${ }^{31}$ S. Viret,${ }^{31}$ Z. Tsamalaidze, ${ }^{32, p}$ C. Autermann, ${ }^{33}$ S. Beranek, ${ }^{33}$ B. Calpas,${ }^{33}$ M. Edelhoff, ${ }^{33}$ L. Feld, ${ }^{33}$ N. Heracleous, ${ }^{33}$ O. Hindrichs, ${ }^{33}$ R. Jussen, ${ }^{33}$ K. Klein, ${ }^{33}$ J. Merz, ${ }^{33}$ A. Ostapchuk,${ }^{33}$ A. Perieanu, ${ }^{33}$ F. Raupach, ${ }^{33}$ J. Sammet, ${ }^{33}$ S. Schael, ${ }^{33}$ D. Sprenger, ${ }^{33}$ H. Weber, ${ }^{33}$ B. Wittmer, ${ }^{33}$ V. Zhukov, ${ }^{33, q}$ M. Ata, ${ }^{34}$ J. Caudron, ${ }^{34}$ E. Dietz-Laursonn, ${ }^{34}$ D. Duchardt,${ }^{34}$ M. Erdmann,${ }^{34}$ R. Fischer, ${ }^{34}$ A. Güth, ${ }^{34}$ T. Hebbeker,${ }^{34}$ C. Heidemann, ${ }^{34}$ K. Hoepfner ${ }^{34}$ D. Klingebiel, ${ }^{34}$ P. Kreuzer, ${ }^{34}$ M. Merschmeyer, ${ }^{34}$ A. Meyer, ${ }^{34}$ M. Olschewski, ${ }^{34}$ K. Padeken, ${ }^{34}$ P. Papacz, ${ }^{34}$ H. Pieta,${ }^{34}$ H. Reithler, ${ }^{34}$ S. A. Schmitz, ${ }^{34}$ L. Sonnenschein, ${ }^{34}$ J. Steggemann, ${ }^{34}$ D. Teyssier, ${ }^{34}$ S. Thüer, ${ }^{34} \mathrm{M}$. Weber, ${ }^{34} \mathrm{M}$. Bontenackels, ${ }^{35} \mathrm{~V}$. Cherepanov, ${ }^{35}$ Y. Erdogan, ${ }^{35}$ G. Flügge,${ }^{35} \mathrm{H}$. Geenen, ${ }^{35}$ M. Geisler, ${ }^{35}$ W. Haj Ahmad, ${ }^{35}$ F. Hoehle, ${ }^{35}$ B. Kargoll, ${ }^{35}$ T. Kress, ${ }^{35}$ Y. Kuessel, ${ }^{35}$ J. Lingemann, ${ }^{35, c}$ A. Nowack, ${ }^{35}$ I. M. Nugent, ${ }^{35}$ L. Perchalla, ${ }^{35}$ O. Pooth,${ }^{35}$ P. Sauerland, ${ }^{35}$ A. Stahl, ${ }^{35}$ M. Aldaya Martin, ${ }^{36}$ I. Asin, ${ }^{36}$ N. Bartosik, ${ }^{36}$ J. Behr, ${ }^{36}$ W. Behrenhoff, ${ }^{36}$ U. Behrens, ${ }^{36}$ M. Bergholz, ${ }^{36, \mathrm{r}}$ A. Bethani, ${ }^{36}$ K. Borras, ${ }^{36}$ A. Burgmeier, ${ }^{36}$ A. Cakir, ${ }^{36}$ L. Calligaris, ${ }^{36}$ A. Campbell, ${ }^{36}$ E. Castro, ${ }^{36}$ F. Costanza, ${ }^{36}$ D. Dammann, ${ }^{36}$ C. Diez Pardos,${ }^{36}$ T. Dorland, ${ }^{36}$ G. Eckerlin, ${ }^{36}$ D. Eckstein, ${ }^{36}$ G. Flucke, ${ }^{36}$ A. Geiser, ${ }^{36}$ I. Glushkov,${ }^{36}$ P. Gunnellini, ${ }^{36}$ S. Habib, ${ }^{36}$ J. Hauk, ${ }^{36}$ G. Hellwig, ${ }^{36}$ H. Jung, ${ }^{36}$ M. Kasemann, ${ }^{36}$ P. Katsas, ${ }^{36}$ C. Kleinwort, ${ }^{36}$ H. Kluge, ${ }^{36}$ A. Knutsson, ${ }^{36}$ M. Krämer, ${ }^{36}$ D. Krücker, ${ }^{36}$ E. Kuznetsova, ${ }^{36}$ W. Lange, ${ }^{36}$ J. Leonard, ${ }^{36}$ W. Lohmann, ${ }^{36, r}$ B. Lutz, ${ }^{36}$ R. Mankel, ${ }^{36}$ I. Marfin, ${ }^{36}$ M. Marienfeld, ${ }^{36}$ I.-A. Melzer-Pellmann, ${ }^{36}$ A. B. Meyer, ${ }^{36}$ J. Mnich,${ }^{36}$ A. Mussgiller, ${ }^{36}$ S. Naumann-Emme, ${ }^{36}$ O. Novgorodova, ${ }^{36}$ F. Nowak, ${ }^{36}$ J. Olzem, ${ }^{36}$ H. Perrey, ${ }^{36}$ A. Petrukhin, ${ }^{36}$ D. Pitzl,${ }^{36}$ A. Raspereza, ${ }^{36}$ P. M. Ribeiro Cipriano, ${ }^{36}$ C. Riedl, ${ }^{36}$ E. Ron, ${ }^{36}$ M. Rosin, ${ }^{36}$ J. Salfeld-Nebgen, ${ }^{36}$ R. Schmidt, ${ }^{36, r}$ T. Schoerner-Sadenius, ${ }^{36}$ N. Sen, ${ }^{36}$ A. Spiridonov, ${ }^{36}$ M. Stein, ${ }^{36}$ R. Walsh, ${ }^{36}$ C. Wissing, ${ }^{36}$ V. Blobel, ${ }^{37}$ H. Enderle, ${ }^{37}$ J. Erfle,${ }^{37}$ U. Gebbert, ${ }^{37}$ M. Görner, ${ }^{37}$ M. Gosselink, ${ }^{37}$ J. Haller,${ }^{37}$ T. Hermanns, ${ }^{37}$ R. S. Höing, ${ }^{37}$ K. Kaschube, ${ }^{37}$ G. Kaussen, ${ }^{37}$ H. Kirschenmann, ${ }^{37}$ R. Klanner, ${ }^{37}$ J. Lange, ${ }^{37}$ T. Peiffer, ${ }^{37}$ N. Pietsch, ${ }^{37}$ D. Rathjens, ${ }^{37}$ C. Sander,${ }^{37}$ H. Schettler, ${ }^{37}$ P. Schleper, ${ }^{37}$ E. Schlieckau, ${ }^{37}$ A. Schmidt, ${ }^{37}$ M. Schröder, ${ }^{37}$ T. Schum, ${ }^{37}$ M. Seidel, ${ }^{37}$ J. Sibille, ${ }^{37, s}$ V. Sola, ${ }^{37}$ H. Stadie, ${ }^{37}$ G. Steinbrück, ${ }^{37}$ J. Thomsen, ${ }^{37}$ L. Vanelderen, ${ }^{37}$ C. Barth,${ }^{38}$ C. Baus, ${ }^{38}$ J. Berger, ${ }^{38}$ C. Böser,${ }^{38}$

T. Chwalek, ${ }^{38}$ W. De Boer ${ }^{38}$ A. Descroix,${ }^{38}$ A. Dierlamm, ${ }^{38}$ M. Feindt, ${ }^{38}$ M. Guthoff, ${ }^{38, c}$ C. Hackstein, ${ }^{38}$

F. Hartmann, ${ }^{38, \mathrm{c}}$ T. Hauth, ${ }^{38, \mathrm{c}}$ M. Heinrich, ${ }^{38}$ H. Held, ${ }^{38}$ K. H. Hoffmann, ${ }^{38}$ U. Husemann, ${ }^{38}$ I. Katkov, ${ }^{38, q}$ J. R. Komaragiri, ${ }^{38}$ P. Lobelle Pardo,${ }^{38}$ D. Martschei, ${ }^{38}$ S. Mueller, ${ }^{38}$ Th. Müller, ${ }^{38}$ M. Niegel, ${ }^{38}$ A. Nürnberg, ${ }^{38}$ O. Oberst, ${ }^{38}$ A. Oehler, ${ }^{38}$ J. Ott, ${ }^{38}$ G. Quast,${ }^{38}$ K. Rabbertz,${ }^{38}$ F. Ratnikov, ${ }^{38}$ N. Ratnikova, ${ }^{38}$ S. Röcker, ${ }^{38}$ F.-P. Schilling, ${ }^{38}$ G. Schott, ${ }^{38}$ H. J. Simonis, ${ }^{38}$ F. M. Stober,${ }^{38}$ D. Troendle, ${ }^{38}$ R. Ulrich, ${ }^{38}$ J. Wagner-Kuhr, ${ }^{38}$ S. Wayand ${ }^{38}$ T. Weiler, ${ }^{38}$ M. Zeise, ${ }^{38}$ G. Anagnostou, ${ }^{39}$ G. Daskalakis, ${ }^{39}$ T. Geralis, ${ }^{39}$ S. Kesisoglou, ${ }^{39}$ A. Kyriakis, ${ }^{39}$ D. Loukas, ${ }^{39}$ A. Markou, ${ }^{39}$ C. Markou, ${ }^{39}$ E. Ntomari, ${ }^{39}$ L. Gouskos, ${ }^{40}$ T. J. Mertzimekis,${ }^{40}$ A. Panagiotou, ${ }^{40}$ N. Saoulidou, ${ }^{40}$ I. Evangelou, ${ }^{41}$ C. Foudas,${ }^{41}$ P. Kokkas, ${ }^{41}$ N. Manthos, ${ }^{41}$ I. Papadopoulos, ${ }^{41}$ G. Bencze,${ }^{42}$ C. Hajdu, ${ }^{42}$ P. Hidas, ${ }^{42}$ D. Horvath,${ }^{42, t}$ F. Sikler, ${ }^{42}$ V. Veszpremi, ${ }^{42}$ G. Vesztergombi, ${ }^{42, u}$ A. J. Zsigmond, ${ }^{42}$ N. Beni, ${ }^{43}$ S. Czellar, ${ }^{43}$ J. Molnar, ${ }^{43}$ J. Palinkas, ${ }^{43}$ Z. Szillasi, ${ }^{43}$ J. Karancsi, ${ }^{44}$ P. Raics,${ }^{44}$ Z. L. Trocsanyi ${ }^{44}$ B. Ujvari, ${ }^{44}$ S. B. Beri, ${ }^{45}$ V. Bhatnagar, ${ }^{45}$ N. Dhingra, ${ }^{45}$ R. Gupta, ${ }^{45}$ M. Kaur, ${ }^{45}$ M.Z. Mehta, ${ }^{45}$ M. Mittal,${ }^{45}$ N. Nishu, ${ }^{45}$ L. K. Saini, ${ }^{45}$ A. Sharma, ${ }^{45}$ J. B. Singh, ${ }^{45}$ Ashok Kumar, ${ }^{46}$ Arun Kumar, ${ }^{46}$ S. Ahuja, ${ }^{46}$ A. Bhardwaj, ${ }^{46}$ B. C. Choudhary, ${ }^{46}$ S. Malhotra, ${ }^{46}$ M. Naimuddin, ${ }^{46}$ K. Ranjan, ${ }^{46}$ P. Saxena,${ }^{46}$ V. Sharma,${ }^{46}$ R. K. Shivpuri ${ }^{46}$ S. Banerjee, ${ }^{47}$ 
S. Bhattacharya,${ }^{47}$ K. Chatterjee, ${ }^{47}$ S. Dutta,${ }^{47}$ B. Gomber, ${ }^{47}$ Sa. Jain, ${ }^{47}$ Sh. Jain, ${ }^{47}$ R. Khurana, ${ }^{47}$ A. Modak,${ }^{47}$ S. Mukherjee, ${ }^{47}$ D. Roy, ${ }^{47}$ S. Sarkar, ${ }^{47}$ M. Sharan,${ }^{47}$ A. Abdulsalam, ${ }^{48}$ D. Dutta, ${ }^{48}$ S. Kailas, ${ }^{48}$ V. Kumar, ${ }^{48}$ A. K. Mohanty, ${ }^{48, \mathrm{c}}$ L. M. Pant, ${ }^{48}$ P. Shukla ${ }^{48}$ T. Aziz,${ }^{49}$ R. M. Chatterjee, ${ }^{49}$ S. Ganguly,${ }^{49}$ M. Guchait, ${ }^{49, v}$ A. Gurtu, ${ }^{49, \mathrm{w}}$ M. Maity, ${ }^{49, \mathrm{x}}$ G. Majumder, ${ }^{49}$ K. Mazumdar, ${ }^{49}$ G. B. Mohanty ${ }^{49}$ B. Parida, ${ }^{49}$ K. Sudhakar, ${ }^{49}$ N. Wickramage, ${ }^{49}$ S. Banerjee, ${ }^{50}$ S. Dugad ${ }^{50}$ H. Arfaei, ${ }^{51, y}$ H. Bakhshiansohi, ${ }^{51}$ S. M. Etesami, ${ }^{51, z}$ A. Fahim, ${ }^{51, y}$ M. Hashemi, ${ }^{51 \text { aa }}$ H. Hesari, ${ }^{51}$ A. Jafari, ${ }^{51}$ M. Khakzad, ${ }^{51}$ M. Mohammadi Najafabadi, ${ }^{51}$ S. Paktinat Mehdiabadi ${ }^{51}$ B. Safarzadeh, ${ }^{51, b b}$ M. Zeinali, ${ }^{51}$ M. Abbrescia, ${ }^{52 a, 52 b}$ L. Barbone, ${ }^{52 a, 52 b}$ C. Calabria, ${ }^{52 a, 52 b, c}$ S. S. Chhibra, ${ }^{52 a, 52 b}$ A. Colaleo, ${ }^{52 a}$ D. Creanza, ${ }^{52 a, 52 c}$ N. De Filippis, ${ }^{52 a, 52 c, c}$ M. De Palma, ${ }^{52 a, 52 b}$ L. Fiore, ${ }^{52 a}$ G. Iaselli, ${ }^{52 a, 52 c}$ G. Maggi, ${ }^{52 a, 52 c}$ M. Maggi, ${ }^{52 a}$ B. Marangelli, ${ }^{52 a, 52 b}$ S. My, ${ }^{52 a, 52 c}$ S. Nuzzo, ${ }^{52 a, 52 b}$ N. Pacifico, ${ }^{52 a}$ A. Pompili, ${ }^{52 a, 52 b}$ G. Pugliese, ${ }^{52 \mathrm{a}, 52 \mathrm{c}}$ G. Selvaggi, ${ }^{52 \mathrm{a}, 52 \mathrm{~b}}$ L. Silvestris, ${ }^{52 \mathrm{a}}$ G. Singh, ${ }^{52 \mathrm{a}, 52 \mathrm{~b}}$ R. Venditti, ${ }^{52 \mathrm{a}, 52 \mathrm{~b}}$ P. Verwilligen, ${ }^{52 \mathrm{a}}$ G. Zito, ${ }^{52 \mathrm{a}}$ G. Abbiendi, ${ }^{53 a}$ A. C. Benvenuti, ${ }^{53 a}$ D. Bonacorsi, ${ }^{53 a, 53 b}$ S. Braibant-Giacomelli, ${ }^{53 a, 53 b}$ L. Brigliadori, ${ }^{53 a, 53 b}$ P. Capiluppi ${ }^{53 a, 53 b}$ A. Castro, ${ }^{53 a, 53 b}$ F. R. Cavallo, ${ }^{53 a}$ M. Cuffiani, ${ }^{53 a, 53 b}$ G. M. Dallavalle, ${ }^{53 a}$ F. Fabbri, ${ }^{53 a}$ A. Fanfani, ${ }^{53 a, 53 b}$ D. Fasanella, ${ }^{53 a, 53 b}$ P. Giacomelli, ${ }^{53 a}$ C. Grandi, ${ }^{53 a}$ L. Guiducci, ${ }^{53 a, 53 b}$ S. Marcellini, ${ }^{53 a}$ G. Masetti, ${ }^{53 a}$ M. Meneghelli, ${ }^{53 a, 53 b, c}$ A. Montanari, ${ }^{53 a}$ F. L. Navarria ${ }^{53 a, 53 b}$ F. Odorici, ${ }^{53 a}$ A. Perrotta, ${ }^{53 a}$ F. Primavera, ${ }^{53 a, 53 b}$ A. M. Rossi, ${ }^{53 a, 53 b}$ T. Rovelli, ${ }^{53 a, 53 b}$ G. P. Siroli, ${ }^{53 a, 53 b}$ N. Tosi, ${ }^{53 a}$ R. Travaglini,,${ }^{53 a, 53 b}$ S. Albergo,${ }^{54 a, 54 b}$ G. Cappello, ${ }^{54 a, 54 b}$ M. Chiorboli, ${ }^{54 a, 54 b}$ S. Costa, ${ }^{54 a, 54 b}$ R. Potenza, ${ }^{54 a, 54 b}$ A. Tricomi, ${ }^{54 a, 54 b}$ C. Tuve, ${ }^{54 a, 54 b}$ G. Barbagli, ${ }^{55 a}$ V. Ciulli,${ }^{55 a, 55 b}$ C. Civinini, ${ }^{55 a}$ R. D’Alessandro, ${ }^{55 a, 55 b}$ E. Focardi, ${ }^{55,55 b}$ S. Frosali, ${ }^{55 a, 55 b}$ E. Gallo, ${ }^{55 a}$ S. Gonzi, ${ }^{55 a, 55 b}$ M. Meschini, ${ }^{55 a}$ S. Paoletti, ${ }^{55 a}$ G. Sguazzoni, ${ }^{55 a}$ A. Tropiano, ${ }^{55 a, 55 b}$ L. Benussi, ${ }^{56}$ S. Bianco, ${ }^{56}$ S. Colafranceschi, ${ }^{56, c c}$ F. Fabbri, ${ }^{56}$ D. Piccolo,${ }^{56}$ P. Fabbricatore,${ }^{57 a}$ R. Musenich, ${ }^{57 a}$ S. Tosi, ${ }^{57 a, 57 b}$ A. Benaglia, ${ }^{58 a}$ F. De Guio, ${ }^{58 a, 58 b}$ L. Di Matteo, ${ }^{58 a, 58 b, c}$ S. Fiorendi, ${ }^{58 a, 58 b}$ S. Gennai, ${ }^{58 a, c}$ A. Ghezzi, ${ }^{58 a, 58 b}$ M. T. Lucchini, ${ }^{58 a, c}$ S. Malvezzi, ${ }^{58 a}$ R. A. Manzoni, ${ }^{58 a, 58 b}$ A. Martelli, ${ }^{58 a, 58 b}$ A. Massironi, ${ }^{58 a, 58 b}$ D. Menasce, ${ }^{58 \mathrm{a}}$ L. Moroni, ${ }^{58 \mathrm{a}}$ M. Paganoni, ${ }^{58 a, 58 b}$ D. Pedrini ${ }^{58 \mathrm{a}}$ S. Ragazzi, ${ }^{58 a, 58 b}$ N. Redaelli, ${ }^{58 \mathrm{a}}$

T. Tabarelli de Fatis, ${ }^{58 a, 58 b}$ S. Buontempo, ${ }^{59 a}$ N. Cavallo, ${ }^{59 a, 59 c}$ A. De Cosa, ${ }^{59 a, 59 b, c}$ O. Dogangun, ${ }^{59 a, 59 b}$ F. Fabozzi, ${ }^{59 a, 59 c}$ A. O. M. Iorio, ${ }^{59 a, 59 b}$ L. Lista, ${ }^{59 a}$ S. Meola,${ }^{59 a, 59 d, c}$ M. Merola, ${ }^{59 a}$ P. Paolucci, ${ }^{59 a, c}$ P. Azzi, ${ }^{60 a}$ N. Bacchetta, ${ }^{60 a, c}$ D. Bisello, ${ }^{60 a, 60 b}$ A. Branca, ${ }^{60 a, 60 b, c}$ R. Carlin, ${ }^{60 a, 60 b}$ P. Checchia, ${ }^{60 \mathrm{a}}$ T. Dorigo, ${ }^{60 \mathrm{a}}$ M. Galanti, ${ }^{60 a, 60 b}$ F. Gasparini, ${ }^{60 a, 60 b}$ U. Gasparini, ${ }^{60 a, 60 b}$ A. Gozzelino, ${ }^{60 a, 60 b}$ K. Kanishchev, ${ }^{60 a, 60 c}$ S. Lacaprara, ${ }^{60 a}$ I. Lazzizzera, ${ }^{60 a, 60 c}$ M. Margoni, ${ }^{60 a, 60 b}$ A. T. Meneguzzo, ${ }^{60 a, 60 b}$ J. Pazzini, ${ }^{60 a, 60 b}$ N. Pozzobon, ${ }^{60 a, 60 b}$ P. Ronchese, ${ }^{60 a, 60 b}$ F. Simonetto,${ }^{60 a, 60 b}$ E. Torassa, ${ }^{60 a}$ M. Tosi, ${ }^{60 a, 60 b}$ S. Vanini, ${ }^{60 a, 60 b}$ P. Zotto, ${ }^{60 a, 60 b}$ A. Zucchetta, ${ }^{60 a, 60 b}$ G. Zumerle, ${ }^{60 a, 60 b}$ M. Gabusi, ${ }^{61 a, 61 b}$ S. P. Ratti, ${ }^{61 \mathrm{a}, 61 \mathrm{~b}}$ C. Riccardi, ${ }^{61 \mathrm{a}, 61 \mathrm{~b}}$ P. Torre, ${ }^{61 \mathrm{a}, 61 \mathrm{~b}}$ P. Vitulo, ${ }^{61 a, 61 b}$ M. Biasini, ${ }^{62 a, 62 b}$ G. M. Bilei, ${ }^{62 a}$ L. Fanò, ${ }^{62 a, 62 b}$ P. Lariccia, ${ }^{62 a, 62 b}$ G. Mantovani, ${ }^{62 a, 62 b}$ M. Menichelli, ${ }^{62 a}$ A. Nappi, ${ }^{62 a, 62 b, a}$ F. Romeo, ${ }^{62 a, 62 b}$ A. Saha, ${ }^{62 a}$ A. Santocchia, ${ }^{62 a, 62 b}$ A. Spiezia, ${ }^{62 a, 62 b}$ S. Taroni, ${ }^{62 a, 62 b}$ P. Azzurri, ${ }^{63 a, 63 c}$ G. Bagliesi, ${ }^{63 a}$ J. Bernardini, ${ }^{63 a}$ T. Boccali, ${ }^{63 a}$ G. Broccolo, ${ }^{63 a, 63 c}$ R. Castaldi, ${ }^{63 a}$ R. T. D’Agnolo ${ }^{63 a, 63 c, c}$ R. Dell'Orso, ${ }^{63 a}$ F. Fiori, ${ }^{63 a, 63 b, c}$ L. Foà, ${ }^{63 a, 63 c}$ A. Giassi, ${ }^{63 a}$ A. Kraan, ${ }^{63 a}$ F. Ligabue, ${ }^{63 a, 63 c}$ T. Lomtadze, ${ }^{63 a}$ L. Martini, ${ }^{63 a, d d}$ A. Messineo ${ }^{63 a, 63 b}$ F. Palla, ${ }^{63 a}$ A. Rizzi, ${ }^{63 a, 63 b}$ A. T. Serban, ${ }^{63 a, e e}$ P. Spagnolo, ${ }^{63 a}$ P. Squillacioti, ${ }^{63 a, c}$ R. Tenchini, ${ }^{63 a}$ G. Tonelli, ${ }^{63 a, 63 b}$ A. Venturi, ${ }^{63 a}$ P. G. Verdini, ${ }^{63 a}$ L. Barone, ${ }^{64 a, 64 b}$ F. Cavallari, ${ }^{64 a}$

D. Del Re, ${ }^{64 a, 64 b}$ M. Diemoz, ${ }^{64 a}$ C. Fanelli, ${ }^{64 a, 64 b}$ M. Grassi, ${ }^{64 a, 64 b, c}$ E. Longo, ${ }^{64 a, 64 b}$ P. Meridiani,,${ }^{64 a, c}$

F. Micheli, ${ }^{64 a, 64 b}$ S. Nourbakhsh, ${ }^{64 a, 64 b}$ G. Organtini, ${ }^{64 a, 64 b}$ R. Paramatti, ${ }^{64 a}$ S. Rahatlou, ${ }^{64 a, 64 b}$ L. Soffi, ${ }^{64 a, 64 b}$

N. Amapane, ${ }^{65 a, 65 b}$ R. Arcidiacono, ${ }^{65 a, 65 \mathrm{c}}$ S. Argiro, ${ }^{65 a, 65 b}$ M. Arneodo, ${ }^{65 a, 65 c}$ C. Biino, ${ }^{65 a}$ N. Cartiglia, ${ }^{65 a}$ S. Casasso, ${ }^{65 a, 65 b}$ M. Costa, ${ }^{65 a, 65 b}$ N. Demaria, ${ }^{65 a}$ C. Mariotti, ${ }^{65 a, c}$ S. Maselli, ${ }^{65 a}$ E. Migliore, ${ }^{65 a, 65 b}$ V. Monaco,${ }^{65 a, 65 b}$

M. Musich, ${ }^{65 a, c}$ M. M. Obertino, ${ }^{65 a, 65 c}$ N. Pastrone, ${ }^{65 a}$ M. Pelliccioni, ${ }^{65 a}$ A. Potenza, ${ }^{65 a, 65 b}$ A. Romero, ${ }^{65 a, 65 b}$ M. Ruspa ${ }^{65 a, 65 c}$ R. Sacchi, ${ }^{65 a, 65 b}$ A. Solano, ${ }^{65 a, 65 b}$ A. Staiano, ${ }^{65 a}$ S. Belforte, ${ }^{66 a}$ V. Candelise,${ }^{66 a, 66 b}$ M. Casarsa, ${ }^{66 a}$ F. Cossutti, ${ }^{66, c}$ G. Della Ricca, ${ }^{66 a, 66 b}$ B. Gobbo, ${ }^{66 a}$ M. Marone, ${ }^{66 a, 66 b, c}$ D. Montanino, ${ }^{66 a, 66 b}$ A. Penzo, ${ }^{66 a}$

A. Schizzi, ${ }^{66 a, 66 b}$ T. Y. Kim, ${ }^{67}$ S. K. Nam, ${ }^{67}$ S. Chang, ${ }^{68}$ D. H. Kim, ${ }^{68}$ G. N. Kim, ${ }^{68}$ D. J. Kong, ${ }^{68}$ H. Park, ${ }^{68}$ D. C. Son,${ }^{68}$ J. Y. Kim, ${ }^{69}$ Zero J. Kim, ${ }^{69}$ S. Song, ${ }^{69}$ S. Choi, ${ }^{70}$ D. Gyun, ${ }^{70}$ B. Hong,${ }^{70}$ M. Jo, ${ }^{70}$ H. Kim, ${ }^{70}$ T. J. Kim, ${ }^{70}$ K. S. Lee, ${ }^{70}$ D. H. Moon,${ }^{70}$ S. K. Park,${ }^{70}$ Y. Roh, ${ }^{70}$ M. Choi,${ }^{71}$ J. H. Kim, ${ }^{71}$ C. Park, ${ }^{71}$ I. C. Park, ${ }^{71}$ S. Park, ${ }^{71}$ G. Ryu, ${ }^{71}$ Y. Choi, ${ }^{72}$ Y. K. Choi,${ }^{72}$ J. Goh ${ }^{72}$ M. S. Kim, ${ }^{72}$ E. Kwon, ${ }^{72}$ B. Lee, ${ }^{72}$ J. Lee, ${ }^{72}$ S. Lee ${ }^{72}$ H. Seo, ${ }^{72}$ I. Yu, ${ }^{72}$ M. J. Bilinskas, ${ }^{73}$ I. Grigelionis, ${ }^{73}$ M. Janulis, ${ }^{73}$ A. Juodagalvis, ${ }^{73}$ H. Castilla-Valdez, ${ }^{74}$ E. De La Cruz-Burelo, ${ }^{74}$ I. Heredia-de La Cruz ${ }^{74}$ R. Lopez-Fernandez,${ }^{74}$ J. Martínez-Ortega ${ }^{74}$ A. Sanchez-Hernandez, ${ }^{74}$

L. M. Villasenor-Cendejas, ${ }^{74}$ S. Carrillo Moreno, ${ }^{75}$ F. Vazquez Valencia, ${ }^{75}$ H. A. Salazar Ibarguen, ${ }^{76}$

E. Casimiro Linares,${ }^{77}$ A. Morelos Pineda, ${ }^{77}$ M. A. Reyes-Santos,${ }^{77}$ D. Krofcheck,${ }^{78}$ A. J. Bell, ${ }^{79}$ P. H. Butler, ${ }^{79}$ R. Doesburg, ${ }^{79}$ S. Reucroft, ${ }^{79}$ H. Silverwood, ${ }^{79}$ M. Ahmad, ${ }^{80}$ M. I. Asghar, ${ }^{80}$ J. Butt, ${ }^{80}$ H. R. Hoorani, ${ }^{80}$ S. Khalid, ${ }^{80}$ 
W. A. Khan, ${ }^{80}$ T. Khurshid ${ }^{80}$ S. Qazi ${ }^{80}$ M. A. Shah, ${ }^{80}$ M. Shoaib,${ }^{80}$ H. Bialkowska, ${ }^{81}$ B. Boimska, ${ }^{81}$ T. Frueboes ${ }^{81}$ M. Górski, ${ }^{81}$ M. Kazana,${ }^{81}$ K. Nawrocki ${ }^{81}$ K. Romanowska-Rybinska ${ }^{81}$ M. Szleper, ${ }^{81}$ G. Wrochna, ${ }^{81}$ P. Zalewski ${ }^{81}$ G. Brona, ${ }^{82}$ K. Bunkowski, ${ }^{82}$ M. Cwiok, ${ }^{82}$ W. Dominik, ${ }^{82}$ K. Doroba, ${ }^{82}$ A. Kalinowski, ${ }^{82}$ M. Konecki, ${ }^{82}$ J. Krolikowski, ${ }^{82}$ M. Misiura, ${ }^{82}$ W. Wolszczak,${ }^{82}$ N. Almeida ${ }^{83}$ P. Bargassa, ${ }^{83}$ A. David ${ }^{83}$ P. Faccioli, ${ }^{83}$ P. G. Ferreira Parracho, ${ }^{83}$ M. Gallinaro, ${ }^{83}$ J. Seixas, ${ }^{83, c}$ J. Varela, ${ }^{83}$ P. Vischia,${ }^{83}$ I. Belotelov,${ }^{84}$ P. Bunin,${ }^{84}$ M. Gavrilenko, ${ }^{84}$ I. Golutvin, ${ }^{84}$ I. Gorbunov, ${ }^{84}$ A. Kamenev, ${ }^{84}$ V. Karjavin,${ }^{84}$ G. Kozlov, ${ }^{84}$ A. Lanev, ${ }^{84}$ A. Malakhov, ${ }^{84}$ P. Moisenz, ${ }^{84}$ V. Palichik, ${ }^{84}$ V. Perelygin, ${ }^{84}$ S. Shmatov, ${ }^{84}$ V. Smirnov, ${ }^{84}$ A. Volodko, ${ }^{84}$ A. Zarubin,${ }^{84}$ S. Evstyukhin, ${ }^{85}$ V. Golovtsov, ${ }^{85}$ Y. Ivanov, ${ }^{85}$ V. Kim,${ }^{85}$ P. Levchenko, ${ }^{85}$ V. Murzin, ${ }^{85}$ V. Oreshkin, ${ }^{85}$ I. Smirnov, ${ }^{85}$ V. Sulimov, ${ }^{85}$ L. Uvarov, ${ }^{85}$ S. Vavilov, ${ }^{85}$ A. Vorobyev, ${ }^{85}$ An. Vorobyev, ${ }^{85}$ Yu. Andreev, ${ }^{86}$ A. Dermenev, ${ }^{86}$ S. Gninenko, ${ }^{86}$ N. Golubev, ${ }^{86}$ M. Kirsanov, ${ }^{86}$ N. Krasnikov, ${ }^{86}$ V. Matveev, ${ }^{86}$ A. Pashenkov, ${ }^{86}$ D. Tlisov, ${ }^{86}$ A. Toropin, ${ }^{86}$ V. Epshteyn, ${ }^{87}$ M. Erofeeva,${ }^{87}$ V. Gavrilov ${ }^{87}$ M. Kossov, ${ }^{87}$ N. Lychkovskaya,${ }^{87}$ V. Popov, ${ }^{87}$ G. Safronov, ${ }^{87}$ S. Semenov, ${ }^{87}$ I. Shreyber ${ }^{87}$ V. Stolin, ${ }^{87}$ E. Vlasov, ${ }^{87}$ A. Zhokin, ${ }^{87}$ A. Belyaev, ${ }^{88}$ E. Boos, ${ }^{88}$ V. Bunichev, ${ }^{88}$ M. Dubinin, ${ }^{88, f}$ L. Dudko, ${ }^{88}$ A. Ershov, ${ }^{88}$ A. Gribushin, ${ }^{88}$ V. Klyukhin, ${ }^{88}$ O. Kodolova, ${ }^{88}$ I. Lokhtin ${ }^{88}$ A. Markina, ${ }^{88}$ S. Obraztsov, ${ }^{88}$ M. Perfilov, ${ }^{88}$ S. Petrushanko, ${ }^{88}$ A. Popov, ${ }^{88}$ L. Sarycheva, ${ }^{88, a}$ V. Savrin,${ }^{88}$ V. Andreev, ${ }^{89}$ M. Azarkin, ${ }^{89}$ I. Dremin, ${ }^{89}$ M. Kirakosyan, ${ }^{89}$ A. Leonidov, ${ }^{89}$ G. Mesyats ${ }^{89}$ S. V. Rusakov, ${ }^{89}$ A. Vinogradov, ${ }^{89}$ I. Azhgirey, ${ }^{90}$ I. Bayshev, ${ }^{90}$ S. Bitioukov,${ }^{90}$ V. Grishin, ${ }^{90, c}$ V. Kachanov, ${ }^{90}$ D. Konstantinov, ${ }^{90}$ V. Krychkine, ${ }^{90}$ V. Petrov, ${ }^{90}$ R. Ryutin, ${ }^{90}$ A. Sobol, ${ }^{90}$ L. Tourtchanovitch, ${ }^{90}$ S. Troshin, ${ }^{90}$ N. Tyurin, ${ }^{90}$ A. Uzunian, ${ }^{90}$ A. Volkov, ${ }^{90}$ P. Adzic,${ }^{91, f f}$ M. Djordjevic,,${ }^{91}$ M. Ekmedzic, ${ }^{91}$ D. Krpic,${ }^{91, f f}$ J. Milosevic, ${ }^{91}$ M. Aguilar-Benitez, ${ }^{92}$ J. Alcaraz Maestre, ${ }^{92}$ P. Arce, ${ }^{92}$ C. Battilana, ${ }^{92}$ E. Calvo, ${ }^{92}$ M. Cerrada, ${ }^{92}$ M. Chamizo Llatas, ${ }^{92}$ N. Colino, ${ }^{92}$ B. De La Cruz, ${ }^{92}$ A. Delgado Peris, ${ }^{92}$ D. Domínguez Vázquez, ${ }^{92}$ C. Fernandez Bedoya ${ }^{92}$ J. P. Fernández Ramos, ${ }^{92}$ A. Ferrando, ${ }^{92}$ J. Flix, ${ }^{92}$ M. C. Fouz, ${ }^{92}$ P. Garcia-Abia, ${ }^{92}$ O. Gonzalez Lopez, ${ }^{92}$ S. Goy Lopez, ${ }^{92}$ J. M. Hernandez, ${ }^{92}$ M. I. Josa, ${ }^{92}$ G. Merino, ${ }^{92}$ J. Puerta Pelayo, ${ }^{92}$ A. Quintario Olmeda, ${ }^{92}$ I. Redondo, ${ }^{92}$ L. Romero, ${ }^{92}$ J. Santaolalla, ${ }^{92}$ M. S. Soares, ${ }^{92}$ C. Willmott, ${ }^{92}$ C. Albajar, ${ }^{93}$ G. Codispoti, ${ }^{93}$ J. F. de Trocóniz, ${ }^{93}$ H. Brun, ${ }^{94}$ J. Cuevas, ${ }^{94}$ J. Fernandez Menendez, ${ }^{94}$ S. Folgueras, ${ }^{94}$ I. Gonzalez Caballero, ${ }^{94}$ L. Lloret Iglesias, ${ }^{94}$ J. Piedra Gomez,${ }^{94}$ J. A. Brochero Cifuentes, ${ }^{95}$ I. J. Cabrillo, ${ }^{95}$ A. Calderon, ${ }^{95}$ S. H. Chuang, ${ }^{95}$ J. Duarte Campderros, ${ }^{95}$ M. Felcini, ${ }^{95, g g}$ M. Fernandez, ${ }^{95}$ G. Gomez, ${ }^{95}$ J. Gonzalez Sanchez, ${ }^{95}$ A. Graziano,,${ }^{95}$ C. Jorda, ${ }^{95}$ A. Lopez Virto, ${ }^{95}$ J. Marco, ${ }^{95}$ R. Marco,${ }^{95}$ C. Martinez Rivero, ${ }^{95}$ F. Matorras, ${ }^{95}$ F. J. Munoz Sanchez, ${ }^{95}$ T. Rodrigo, ${ }^{95}$ A. Y. Rodríguez-Marrero, ${ }^{95}$ A. Ruiz-Jimeno, ${ }^{95}$ L. Scodellaro, ${ }^{95}$ I. Vila, ${ }^{95}$ R. Vilar Cortabitarte, ${ }^{95}$ D. Abbaneo, ${ }^{96}$ E. Auffray, ${ }^{96}$ G. Auzinger, ${ }^{96}$ M. Bachtis, ${ }^{96}$ P. Baillon, ${ }^{96}$ A. H. Ball, ${ }^{96}$ D. Barney, ${ }^{96}$ J. Bendavid,${ }^{96}$ J. F. Benitez,${ }^{96}$ C. Bernet ${ }^{96, g}$ G. Bianchi, ${ }^{96}$ P. Bloch, ${ }^{96}$ A. Bocci,${ }^{96}$ A. Bonato, ${ }^{96}$ C. Botta,${ }^{96}$ H. Breuker, ${ }^{96}$ T. Camporesi,${ }^{96}$ G. Cerminara,${ }^{96}$ T. Christiansen, ${ }^{96}$

J. A. Coarasa Perez,${ }^{96}$ D. d'Enterria, ${ }^{96}$ A. Dabrowski,${ }^{96}$ A. De Roeck, ${ }^{96}$ S. De Visscher,${ }^{96}$ S. Di Guida,${ }^{96}$

M. Dobson, ${ }^{96}$ N. Dupont-Sagorin, ${ }^{96}$ A. Elliott-Peisert, ${ }^{96}$ J. Eugster, ${ }^{96}$ B. Frisch, ${ }^{96}$ W. Funk, ${ }^{96}$ G. Georgiou,${ }^{96}$ M. Giffels, ${ }^{96}$ D. Gigi, ${ }^{96}$ K. Gill, ${ }^{96}$ D. Giordano,,${ }^{96}$ M. Girone, ${ }^{96}$ M. Giunta,${ }^{96}$ F. Glege, ${ }^{96}$ R. Gomez-Reino Garrido, ${ }^{96}$ P. Govoni, ${ }^{96}$ S. Gowdy, ${ }^{96}$ R. Guida, ${ }^{96}$ J. Hammer, ${ }^{96}$ M. Hansen, ${ }^{96}$ P. Harris,,${ }^{96}$ C. Hartl, ${ }^{96}$ J. Harvey, ${ }^{96}$ B. Hegner, ${ }^{96}$ A. Hinzmann, ${ }^{96}$ V. Innocente, ${ }^{96}$ P. Janot,${ }^{96}$ K. Kaadze, ${ }^{96}$ E. Karavakis, ${ }^{96}$ K. Kousouris, ${ }^{96}$ K. Krajczar, ${ }^{96}$ P. Lecoq, ${ }^{96}$ Y.-J. Lee, ${ }^{96}$ P. Lenzi,${ }^{96}$ C. Lourenço, ${ }^{96}$ N. Magini,${ }^{96}$ T. Mäki, ${ }^{96}$ M. Malberti, ${ }^{96}$ L. Malgeri, ${ }^{96}$ M. Mannelli, ${ }^{96}$ L. Masetti, ${ }^{96}$ F. Meijers, ${ }^{96}$ S. Mersi,${ }^{96}$ E. Meschi,${ }^{96}$ R. Moser, ${ }^{96}$ M. Mulders,${ }^{96}$ P. Musella, ${ }^{96}$ E. Nesvold,${ }^{96}$ L. Orsini,${ }^{96}$ E. Palencia Cortezon, ${ }^{96}$ E. Perez,${ }^{96}$ L. Perrozzi, ${ }^{96}$ A. Petrilli, ${ }^{96}$ A. Pfeiffer, ${ }^{96}$ M. Pierini, ${ }^{96}$ M. Pimiä, ${ }^{96}$ D. Piparo, ${ }^{96}$ G. Polese, ${ }^{96}$ L. Quertenmont, ${ }^{96}$ A. Racz, ${ }^{96}$ W. Reece, ${ }^{96}$ J. Rodrigues Antunes,${ }^{96}$ G. Rolandi, ${ }^{96, h h}$ C. Rovelli, ${ }^{96, i i}$ M. Rovere,${ }^{96}$ H. Sakulin, ${ }^{96}$ F. Santanastasio, ${ }^{96}$ C. Schäfer, ${ }^{96}$ C. Schwick, ${ }^{96}$ I. Segoni, ${ }^{96}$ S. Sekmen, ${ }^{96}$ A. Sharma, ${ }^{96}$ P. Siegrist,${ }^{96}$ P. Silva, ${ }^{96}$ M. Simon, ${ }^{96}$ P. Sphicas, ${ }^{96, j j}$ D. Spiga ${ }^{96}$ A. Tsirou,${ }^{96}$ G. I. Veres, ${ }^{96, u}$ J. R. Vlimant,${ }^{96}$ H. K. Wöhri, ${ }^{96}$ S. D. Worm, ${ }^{96, k k}$ W. D. Zeuner, ${ }^{96}$ W. Bertl, ${ }^{97}$ K. Deiters,${ }^{97}$ W. Erdmann,,${ }^{97}$ K. Gabathuler,${ }^{97}$ R. Horisberger, ${ }^{97}$ Q. Ingram, ${ }^{97}$ H. C. Kaestli, ${ }^{97}$ S. König, ${ }^{97}$ D. Kotlinski, ${ }^{97}$ U. Langenegger, ${ }^{97}$ F. Meier,${ }^{97}$ D. Renker, ${ }^{97}$ T. Rohe, ${ }^{97}$ F. Bachmair, ${ }^{98}$ L. Bäni,${ }^{98}$ P. Bortignon,,${ }^{98}$ M. A. Buchmann,,${ }^{98}$ B. Casal,${ }^{98}$ N. Chanon, ${ }^{98}$ A. Deisher, ${ }^{98}$ G. Dissertori, ${ }^{98}$ M. Dittmar,${ }^{98}$ M. Donegà, ${ }^{98}$ M. Dünser, ${ }^{98}$ P. Eller, ${ }^{98}$ K. Freudenreich,${ }^{98}$ C. Grab, ${ }^{98}$ D. Hits, ${ }^{98}$ P. Lecomte, ${ }^{98}$ W. Lustermann, ${ }^{98}$ A. C. Marini, ${ }^{98}$ P. Martinez Ruiz del Arbol, ${ }^{98}$ N. Mohr, ${ }^{98}$ F. Moortgat, ${ }^{98}$ C. Nägeli, ${ }^{98,11}$ P. Nef, ${ }^{98}$ F. Nessi-Tedaldi, ${ }^{98}$ F. Pandolfi, ${ }^{98}$ L. Pape,${ }^{98}$ F. Pauss,${ }^{98}$ M. Peruzzi,${ }^{98}$ F. J. Ronga,${ }^{98}$ M. Rossini, ${ }^{98}$ L. Sala, ${ }^{98}$ A. K. Sanchez, ${ }^{98}$ A. Starodumov, ${ }^{98, m m}$ B. Stieger, ${ }^{98}$ M. Takahashi, ${ }^{98}$ L. Tauscher, ${ }^{98, a}$ A. Thea,${ }^{98}$ K. Theofilatos, ${ }^{98}$ D. Treille, ${ }^{98}$ C. Urscheler, ${ }^{98}$ R. Wallny, ${ }^{98}$ H. A. Weber,${ }^{98}$ L. Wehrli, ${ }^{98}$ C. Amsler,${ }^{99, n n}$ V. Chiochia ${ }^{99}$ C. Favaro, ${ }^{99}$ M. Ivova Rikova, ${ }^{99}$ B. Kilminster, ${ }^{99}$ B. Millan Mejias, ${ }^{99}$ P. Otiougova, ${ }^{99}$ P. Robmann, ${ }^{99}$ H. Snoek, ${ }^{99}$ S. Tupputi, ${ }^{99}$ M. Verzetti, ${ }^{99}$ M. Cardaci, ${ }^{100}$ Y. H. Chang, ${ }^{100}$ K. H. Chen, ${ }^{100}$ C. Ferro, ${ }^{100}$ C. M. Kuo, ${ }^{100}$ 
S. W. Li, ${ }^{100}$ W. Lin,${ }^{100}$ Y. J. Lu, ${ }^{100}$ A. P. Singh, ${ }^{100}$ R. Volpe, ${ }^{100}$ S. S. Yu, ${ }^{100}$ P. Bartalini, ${ }^{101}$ P. Chang, ${ }^{101}$ Y. H. Chang, ${ }^{101}$ Y. W. Chang, ${ }^{101}$ Y. Chao, ${ }^{101}$ K. F. Chen,${ }^{101}$ C. Dietz,${ }^{101}$ U. Grundler, ${ }^{101}$ W.-S. Hou, ${ }^{101}$ Y. Hsiung,,${ }^{101}$ K. Y. Kao ${ }_{101}$ Y. J. Lei, ${ }^{101}$ R.-S. Lu, ${ }^{101}$ D. Majumder ${ }^{101}$ E. Petrakou, ${ }^{101}$ X. Shi, ${ }^{101}$ J. G. Shiu, ${ }^{101}$ Y. M. Tzeng, ${ }^{101}$ X. Wan, ${ }^{101}$ M. Wang, ${ }^{101}$ B. Asavapibhop, ${ }^{102}$ E. Simili, ${ }^{102}$ N. Srimanobhas, ${ }^{102}$ N. Suwonjandee, ${ }^{102}$ A. Adiguzel,,${ }^{103}$ M. N. Bakirci, ${ }^{103, \text { oo }}$ S. Cerci, ${ }^{103, p p}$ C. Dozen, ${ }^{103}$ I. Dumanoglu, ${ }^{103}$ E. Eskut, ${ }^{103}$ S. Girgis, ${ }^{103}$ G. Gokbulut, ${ }^{103}$ E. Gurpinar, ${ }^{103}$ I. Hos, ${ }^{103}$ E. E. Kangal, ${ }^{103}$ T. Karaman, ${ }^{103}$ G. Karapinar, ${ }^{103, q q}$ A. Kayis Topaksu, ${ }^{103}$ G. Onengut, ${ }^{103}$ K. Ozdemir, ${ }^{103}$ S. Ozturk, ${ }^{103, \text { rr }}$ A. Polatoz, ${ }^{103}$ K. Sogut, ${ }^{103, \text { ss }}$ D. Sunar Cerci, ${ }^{103, p p}$ B. Tali, ${ }^{103, p p}$ H. Topakli, ${ }^{103, o o}$ M. Vergili, ${ }^{103}$ I. V. Akin, ${ }^{104}$ T. Aliev, ${ }^{104}$ B. Bilin, ${ }^{104}$ S. Bilmis, ${ }^{104}$ M. Deniz, ${ }^{104}$ H. Gamsizkan, ${ }^{104}$ A. M. Guler, ${ }^{104}$ K. Ocalan, ${ }^{104}$ A. Ozpineci, ${ }^{104}$ M. Serin, ${ }^{104}$ R. Sever, ${ }^{104}$ U. E. Surat,${ }^{104}$ M. Yalvac, ${ }^{104}$ M. Zeyrek, ${ }^{104}$ E. Gülmez, ${ }^{105}$ B. Isildak, ${ }^{105, t \mathrm{tt}}$ M. Kaya, ${ }^{105, \text { uu }}$ O. Kaya, ${ }^{105, \text { uu }}$ S. Ozkorucuklu, ${ }^{105, v v}$ N. Sonmez, ${ }^{105, w w}$ H. Bahtiyar, ${ }^{106, x x}$ E. Barlas, ${ }^{106}$ K. Cankocak, ${ }^{106}$ Y. O. Günaydin, ${ }^{106, y y}$ F. I. Vardarl1, ${ }^{106}$ M. Yücel,,${ }^{106}$ L. Levchuk,,${ }^{107}$ J. J. Brooke, ${ }^{108}$ E. Clement, ${ }^{108}$ D. Cussans,${ }^{108}$ H. Flacher, ${ }^{108}$ R. Frazier, ${ }^{108}$ J. Goldstein, ${ }^{108}$ M. Grimes, ${ }^{108}$ G. P. Heath, ${ }^{108}$ H. F. Heath, ${ }^{108}$ L. Kreczko, ${ }^{108}$ S. Metson, ${ }^{108}$ D. M. Newbold, ${ }^{108, k k}$ K. Nirunpong, ${ }^{108}$ A. Poll, ${ }^{108}$ S. Senkin, ${ }^{108}$ V. J. Smith, ${ }^{108}$ T. Williams, ${ }^{108}$ L. Basso, ${ }^{109, z z}$ K. W. Bell, ${ }^{109}$ A. Belyaev, ${ }^{109, z z}$ C. Brew, ${ }^{109}$ R. M. Brown, ${ }^{109}$ D. J. A. Cockerill,,${ }^{109}$ J. A. Coughlan, ${ }^{109}$ K. Harder, ${ }^{109}$ S. Harper, ${ }^{109}$ J. Jackson, ${ }^{109}$ B. W. Kennedy, ${ }^{109}$ E. Olaiya, ${ }^{109}$ D. Petyt, ${ }^{109}$

B. C. Radburn-Smith, ${ }^{109}$ C. H. Shepherd-Themistocleous, ${ }^{109}$ I. R. Tomalin, ${ }^{109}$ W. J. Womersley, ${ }^{109}$ R. Bainbridge, ${ }^{110}$ G. Ball, ${ }^{110}$ R. Beuselinck, ${ }^{110}$ O. Buchmuller, ${ }^{110}$ D. Colling, ${ }^{110}$ N. Cripps,,${ }^{110}$ M. Cutajar, ${ }^{110}$ P. Dauncey, ${ }^{110}$

G. Davies, ${ }^{110}$ M. Della Negra, ${ }^{110}$ W. Ferguson, ${ }^{110}$ J. Fulcher,${ }^{10}$ D. Futyan, ${ }^{110}$ A. Gilbert, ${ }^{10}$ A. Guneratne Bryer, ${ }^{110}$ G. Hall, ${ }^{110}$ Z. Hatherell, ${ }^{110}$ J. Hays, ${ }^{110}$ G. Iles, ${ }^{110}$ M. Jarvis, ${ }^{110}$ G. Karapostoli, ${ }^{110}$ M. Kenzie,${ }^{110}$ L. Lyons, ${ }^{110}$ A.-M. Magnan, ${ }^{110}$ J. Marrouche, ${ }^{110}$ B. Mathias, ${ }^{10}$ R. Nandi,,${ }^{110}$ J. Nash, ${ }^{110}$ A. Nikitenko, ${ }^{110, m m}$ J. Pela, ${ }^{110}$ M. Pesaresi, ${ }^{110}$ K. Petridis, ${ }^{110}$ M. Pioppi,,${ }^{110 \text {,aaa }}$ D. M. Raymond, ${ }^{110}$ S. Rogerson, ${ }^{110}$ A. Rose,${ }^{110}$ C. Seez,${ }^{110}$ P. Sharp, ${ }^{110, a}$ A. Sparrow ${ }^{110}$ M. Stoye,${ }^{110}$ A. Tapper,${ }^{110}$ M. Vazquez Acosta, ${ }^{110}$ T. Virdee, ${ }^{110}$ S. Wakefield, ${ }^{110}$ N. Wardle, ${ }^{110}$ T. Whyntie, ${ }^{110}$ M. Chadwick, ${ }^{111}$ J. E. Cole, ${ }^{111}$ P. R. Hobson, ${ }^{111}$ A. Khan, ${ }^{111}$ P. Kyberd, ${ }^{111}$ D. Leggat, ${ }^{111}$ D. Leslie, ${ }^{111}$ W. Martin, ${ }^{111}$ I. D. Reid, ${ }^{111}$ P. Symonds, ${ }^{111}$ L. Teodorescu, ${ }^{111}$ M. Turner, ${ }^{111}$ K. Hatakeyama, ${ }^{112}$ H. Liu, ${ }^{112}$ T. Scarborough, ${ }^{112}$ O. Charaf, ${ }^{113}$ S. I. Cooper, ${ }^{113}$ C. Henderson, ${ }^{113}$ P. Rumerio, ${ }^{113}$ A. Avetisyan, ${ }^{114}$ T. Bose,${ }^{114}$ C. Fantasia, ${ }^{114}$ A. Heister, ${ }^{114}$ J. St. John, ${ }^{114}$ P. Lawson, ${ }^{114}$ D. Lazic, ${ }^{114}$ J. Rohlf, ${ }^{114}$ D. Sperka, ${ }^{114}$ L. Sulak, ${ }^{114}$ J. Alimena, ${ }^{115}$ S. Bhattacharya, ${ }^{115}$ G. Christopher, ${ }^{115}$ D. Cutts, ${ }^{115}$ Z. Demiragli, ${ }^{115}$ A. Ferapontov, ${ }^{115}$ A. Garabedian, ${ }^{115}$ U. Heintz, ${ }^{115}$ S. Jabeen, ${ }^{115}$ G. Kukartsev, ${ }^{115}$ E. Laird, ${ }^{115}$ G. Landsberg, ${ }^{115}$ M. Luk, ${ }^{115}$ M. Narain, ${ }^{115}$ M. Segala, ${ }^{115}$ T. Sinthuprasith, ${ }^{115}$ T. Speer, ${ }^{115}$ R. Breedon, ${ }^{116}$ G. Breto, ${ }^{116}$

M. Calderon De La Barca Sanchez, ${ }^{116}$ M. Caulfield, ${ }^{116}$ S. Chauhan, ${ }^{116}$ M. Chertok, ${ }^{116}$ J. Conway, ${ }^{116}$ R. Conway, ${ }^{116}$ P. T. Cox, ${ }^{116}$ J. Dolen, ${ }^{116}$ R. Erbacher,${ }^{116}$ M. Gardner, ${ }^{116}$ R. Houtz, ${ }^{116}$ W. Ko, ${ }^{116}$ A. Kopecky, ${ }^{116}$ R. Lander, ${ }^{116}$ O. Mall, ${ }^{116}$ T. Miceli, ${ }^{116}$ R. Nelson, ${ }^{116}$ D. Pellett, ${ }^{116}$ F. Ricci-Tam, ${ }^{116}$ B. Rutherford, ${ }^{116}$ M. Searle, ${ }^{116}$ J. Smith, ${ }^{116}$ M. Squires, ${ }^{116}$ M. Tripathi, ${ }^{116}$ R. Vasquez Sierra, ${ }^{116}$ R. Yohay, ${ }^{116}$ V. Andreev ${ }^{117}$ D. Cline, ${ }^{117}$ R. Cousins, ${ }^{117}$ J. Duris, ${ }^{117}$ S. Erhan, ${ }^{117}$ P. Everaerts, ${ }^{117}$ C. Farrell, ${ }^{117}$ J. Hauser, ${ }^{117}$ M. Ignatenko, ${ }^{117}$ C. Jarvis, ${ }^{117}$ G. Rakness, ${ }^{117}$ P. Schlein, ${ }^{117, a}$ P. Traczyk, ${ }^{117}$ V. Valuev, ${ }^{117}$ M. Weber, ${ }^{117}$ J. Babb, ${ }^{118}$ R. Clare, ${ }^{118}$ M. E. Dinardo, ${ }^{118}$ J. Ellison, ${ }^{118}$ J. W. Gary, ${ }^{118}$ F. Giordano, ${ }^{118}$ G. Hanson, ${ }^{118}$ H. Liu, ${ }^{118}$ O. R. Long, ${ }^{118}$ A. Luthra, ${ }^{118}$ H. Nguyen, ${ }^{118}$ S. Paramesvaran, ${ }^{118}$ J. Sturdy, ${ }^{118}$ S. Sumowidagdo, ${ }^{118}$ R. Wilken, ${ }^{118}$ S. Wimpenny, ${ }^{118}$ W. Andrews, ${ }^{119}$ J. G. Branson, ${ }^{119}$ G. B. Cerati, ${ }^{119}$ S. Cittolin, ${ }^{119}$ D. Evans, ${ }^{119}$ A. Holzner, ${ }^{19}$ R. Kelley, ${ }^{119}$ M. Lebourgeois, ${ }^{119}$ J. Letts, ${ }^{119}$ I. Macneill, ${ }^{119}$ B. Mangano, ${ }^{119}$ S. Padhi, ${ }^{119}$ C. Palmer,${ }^{19}$ G. Petrucciani, ${ }^{119}$ M. Pieri, ${ }^{119}$ M. Sani, ${ }^{119}$ V. Sharma, ${ }^{119}$ S. Simon, ${ }^{119}$ E. Sudano, ${ }^{119}$ M. Tadel,${ }^{119}$ Y. Tu, ${ }^{119}$ A. Vartak, ${ }^{119}$ S. Wasserbaech,,${ }^{11, \text { bbb }}$ F. Würthwein, ${ }^{119}$ A. Yagil, ${ }^{119}$ J. Yoo, ${ }^{119}$ D. Barge,${ }^{120}$ R. Bellan, ${ }^{120}$ C. Campagnari, ${ }^{120}$ M. D’ Alfonso, ${ }^{120}$ T. Danielson, ${ }^{120}$ K. Flowers, ${ }^{120}$ P. Geffert, ${ }^{120}$ C. George, ${ }^{120}$ F. Golf,,${ }^{120}$ J. Incandela, ${ }^{120}$ C. Justus, ${ }^{120}$ P. Kalavase, ${ }^{120}$ D. Kovalskyi, ${ }^{120}$ V. Krutelyov, ${ }^{120}$ S. Lowette, ${ }^{120}$ R. Magaña Villalba, ${ }^{120}$ N. Mccoll, ${ }^{120}$ V. Pavlunin, ${ }^{120}$ J. Ribnik, ${ }^{120}$ J. Richman, ${ }^{120}$ R. Rossin, ${ }^{120}$ D. Stuart,,${ }^{120}$ W. To, ${ }^{120}$ C. West,${ }^{120}$ A. Apresyan, ${ }^{121}$ A. Bornheim, ${ }^{121}$ Y. Chen, ${ }^{121}$ E. Di Marco, ${ }^{121}$ J. Duarte, ${ }^{121}$ M. Gataullin, ${ }^{121}$ Y. Ma, ${ }^{121}$ A. Mott, ${ }^{121}$ H. B. Newman, ${ }^{121}$ C. Rogan, ${ }^{121}$ M. Spiropulu, ${ }^{121}$ V. Timciuc, ${ }^{121}$ J. Veverka, ${ }^{121}$ R. Wilkinson, ${ }^{121}$ S. Xie, ${ }^{121}$ Y. Yang, ${ }^{121}$ R. Y. Zhu, ${ }^{121}$ V. Azzolini, ${ }^{122}$ A. Calamba, ${ }^{122}$ R. Carroll, ${ }^{122}$ T. Ferguson, ${ }^{122}$ Y. Iiyama, ${ }^{122}$ D. W. Jang, ${ }^{122}$ Y. F. Liu, ${ }^{122}$ M. Paulini, ${ }^{122}$ H. Vogel, ${ }^{122}$ I. Vorobiev, ${ }^{122}$ J. P. Cumalat, ${ }^{123}$ B. R. Drell, ${ }^{123}$ W. T. Ford, ${ }^{123}$ A. Gaz, ${ }^{123}$ E. Luiggi Lopez, ${ }^{123}$ J. G. Smith, ${ }^{123}$ K. Stenson, ${ }^{123}$ K. A. Ulmer, ${ }^{123}$ S. R. Wagner, ${ }^{123}$ J. Alexander, ${ }^{124}$ A. Chatterjee, ${ }^{124}$ N. Eggert, ${ }^{124}$ L. K. Gibbons, ${ }^{124}$ B. Heltsley, ${ }^{124}$ W. Hopkins, ${ }^{124}$ A. Khukhunaishvili, ${ }^{124}$ B. Kreis, ${ }^{124}$ N. Mirman, ${ }^{124}$ G. Nicolas Kaufman, ${ }^{124}$ J. R. Patterson, ${ }^{124}$ A. Ryd,${ }^{124}$ E. Salvati, ${ }^{124}$ W. Sun, ${ }^{124}$ W. D. Teo, ${ }^{124}$ J. Thom, ${ }^{124}$ J. Thompson, ${ }^{124}$ J. Tucker, ${ }^{124}$ Y. Weng,,${ }^{124}$ 
L. Winstrom, ${ }^{124}$ P. Wittich, ${ }^{124}$ D. Winn, ${ }^{125}$ S. Abdullin, ${ }^{126}$ M. Albrow, ${ }^{126}$ J. Anderson, ${ }^{126}$ G. Apollinari, ${ }^{126}$ L. A. T. Bauerdick, ${ }^{126}$ A. Beretvas, ${ }^{126}$ J. Berryhill, ${ }^{126}$ P. C. Bhat, ${ }^{126}$ K. Burkett, ${ }^{126}$ J. N. Butler, ${ }^{126}$ V. Chetluru, ${ }^{126}$ H. W. K. Cheung, ${ }^{126}$ F. Chlebana, ${ }^{126}$ V. D. Elvira, ${ }^{126}$ I. Fisk, ${ }^{126}$ J. Freeman, ${ }^{126}$ Y. Gao, ${ }^{126}$ D. Green, ${ }^{126}$ O. Gutsche,${ }^{126}$ J. Hanlon, ${ }^{126}$ R. M. Harris, ${ }^{126}$ J. Hirschauer, ${ }^{126}$ B. Hooberman, ${ }^{126}$ S. Jindariani, ${ }^{126}$ M. Johnson, ${ }^{126}$ U. Joshi, ${ }^{126}$

B. Klima, ${ }^{126}$ S. Kunori, ${ }^{126}$ S. Kwan, ${ }^{126}$ C. Leonidopoulos, ${ }^{126, c c c}$ J. Linacre, ${ }^{126}$ D. Lincoln, ${ }^{126}$ R. Lipton, ${ }^{126}$ J. Lykken, ${ }^{126}$ K. Maeshima, ${ }^{126}$ J. M. Marraffino, ${ }^{126}$ V. I. Martinez Outschoorn, ${ }^{126}$ S. Maruyama, ${ }^{126}$ D. Mason, ${ }^{126}$ P. McBride, ${ }^{126}$ K. Mishra, ${ }^{126}$ S. Mrenna, ${ }^{126}$ Y. Musienko, ${ }^{126, d d d}$ C. Newman-Holmes, ${ }^{126}$ V. O’Dell, ${ }^{126}$ E. Sexton-Kennedy, ${ }^{126}$ S. Sharma, ${ }^{126}$ W. J. Spalding, ${ }^{126}$ L. Spiegel, ${ }^{126}$ L. Taylor, ${ }^{126}$ S. Tkaczyk, ${ }^{126}$ N. V. Tran, ${ }^{126}$ L. Uplegger, ${ }^{126}$ E. W. Vaandering, ${ }^{126}$ R. Vidal,${ }^{126}$ J. Whitmore, ${ }^{126}$ W. Wu, ${ }^{126}$ F. Yang, ${ }^{126}$ J. C. Yun, ${ }^{126}$ D. Acosta, ${ }^{127}$ P. Avery, ${ }^{127}$ D. Bourilkov, ${ }^{127}$ M. Chen, ${ }^{127}$ T. Cheng, ${ }^{127}$ S. Das, ${ }^{127}$ M. De Gruttola, ${ }^{127}$ G. P. Di Giovanni, ${ }^{127}$ D. Dobur, ${ }^{127}$ A. Drozdetskiy, ${ }^{127}$ R. D. Field, ${ }^{127}$ M. Fisher, ${ }^{127}$ Y. Fu, ${ }^{127}$ I. K. Furic, ${ }^{127}$ J. Gartner, ${ }^{127}$ J. Hugon, ${ }^{127}$ B. Kim, ${ }^{127}$ J. Konigsberg, ${ }^{127}$ A. Korytov ${ }^{127}$ A. Kropivnitskaya, ${ }^{127}$ T. Kypreos, ${ }^{127}$ J. F. Low ${ }^{127}$ K. Matchev, ${ }^{127}$ P. Milenovic, ${ }^{127, \text { eee }}$ G. Mitselmakher, ${ }^{127}$ L. Muniz, ${ }^{127}$ R. Remington, ${ }^{127}$ A. Rinkevicius, ${ }^{127}$ N. Skhirtladze, ${ }^{127}$ M. Snowball, ${ }^{127}$ J. Yelton, ${ }^{127}$ M. Zakaria, ${ }^{127}$ V. Gaultney, ${ }^{128}$ S. Hewamanage, ${ }^{128}$ L. M. Lebolo, ${ }^{128}$ S. Linn, ${ }^{128}$ P. Markowitz, ${ }^{128}$ G. Martinez, ${ }^{128}$ J. L. Rodriguez, ${ }^{128}$ T. Adams, ${ }^{129}$ A. Askew, ${ }^{129}$ J. Bochenek, ${ }^{129}$ J. Chen, ${ }^{129}$ B. Diamond, ${ }^{129}$ S. V. Gleyzer, ${ }^{129}$ J. Haas, ${ }^{129}$ S. Hagopian, ${ }^{129}$ V. Hagopian, ${ }^{129}$ M. Jenkins, ${ }^{129}$ K. F. Johnson, ${ }^{129}$ H. Prosper ${ }^{129}$ V. Veeraraghavan, ${ }^{129}$ M. Weinberg, ${ }^{129}$ M. M. Baarmand, ${ }^{130}$ B. Dorney, ${ }^{130}$ M. Hohlmann, ${ }^{130}$ H. Kalakhety, ${ }^{130}$ I. Vodopiyanov, ${ }^{130}$ F. Yumiceva, ${ }^{130}$ M. R. Adams, ${ }^{131}$ L. Apanasevich, ${ }^{131}$ Y. Bai, ${ }^{131}$ V. E. Bazterra, ${ }^{131}$ R. R. Betts, ${ }^{131}$ I. Bucinskaite, ${ }^{131}$ J. Callner, ${ }^{131}$ R. Cavanaugh, ${ }^{131}$ O. Evdokimov, ${ }^{131}$ L. Gauthier, ${ }^{131}$ C. E. Gerber, ${ }^{131}$ D. J. Hofman, ${ }^{131}$ S. Khalatyan, ${ }^{131}$ F. Lacroix,${ }^{131}$ C. O’Brien, ${ }^{131}$ C. Silkworth, ${ }^{131}$ D. Strom, ${ }^{131}$ P. Turner, ${ }^{131}$ N. Varelas, ${ }^{131}$ U. Akgun, ${ }^{132}$ E. A. Albayrak, ${ }^{132}$ B. Bilki, ${ }^{132, \text { fff }}$ W. Clarida, ${ }^{132}$ K. Dilsiz, ${ }^{132}$ F. Duru, ${ }^{132}$ S. Griffiths, ${ }^{132}$ J.-P. Merlo, ${ }^{132}$ H. Mermerkaya, ${ }^{132, \text { ggg }}$ A. Mestvirishvili, ${ }^{132}$ A. Moeller, ${ }^{132}$ J. Nachtman, ${ }^{132}$ C. R. Newsom, ${ }^{132}$ E. Norbeck, ${ }^{132}$ H. Ogul, ${ }^{132}$ Y. Onel, ${ }^{132}$ F. Ozok, ${ }^{132, x x}$ S. Sen, ${ }^{132}$ P. Tan, ${ }^{132}$ E. Tiras, ${ }^{132}$ J. Wetzel, ${ }^{132}$ T. Yetkin, ${ }^{132}$ K. Yi, ${ }^{132}$ B. A. Barnett, ${ }^{133}$ B. Blumenfeld, ${ }^{133}$ S. Bolognesi, ${ }^{133}$ D. Fehling, ${ }^{133}$ G. Giurgiu, ${ }^{133}$ A. V. Gritsan, ${ }^{133}$ Z. J. Guo, ${ }^{133}$ G. Hu, ${ }^{133}$ P. Maksimovic, ${ }^{133}$ M. Swartz,${ }^{133}$ A. Whitbeck, ${ }^{133}$ P. Baringer, ${ }^{134}$ A. Bean, ${ }^{134}$ G. Benelli, ${ }^{134}$ R. P. Kenny Iii, ${ }^{134}$ M. Murray, ${ }^{134}$ D. Noonan, ${ }^{134}$ S. Sanders, ${ }^{134}$ R. Stringer, ${ }^{134}$ G. Tinti, ${ }^{134}$ J. S. Wood, ${ }^{134}$ A. F. Barfuss, ${ }^{135}$ T. Bolton, ${ }^{135}$ I. Chakaberia, ${ }^{135}$ A. Ivanov, ${ }^{135}$ S. Khalil, ${ }^{135}$ M. Makouski, ${ }^{135}$ Y. Maravin, ${ }^{135}$ S. Shrestha, ${ }^{135}$ I. Svintradze, ${ }^{135}$ J. Gronberg, ${ }^{136}$ D. Lange, ${ }^{136}$ F. Rebassoo, ${ }^{136}$ D. Wright, ${ }^{136}$ A. Baden, ${ }^{137}$ B. Calvert, ${ }^{137}$ S. C. Eno, ${ }^{137}$ J. A. Gomez, ${ }^{137}$ N. J. Hadley, ${ }^{137}$ R. G. Kellogg, ${ }^{137}$ M. Kirn, ${ }^{137}$ T. Kolberg, ${ }^{137}$ Y. Lu, ${ }^{137}$ M. Marionneau, ${ }^{137}$ A. C. Mignerey, ${ }^{137}$ K. Pedro, ${ }^{137}$ A. Peterman, ${ }^{137}$ A. Skuja, ${ }^{137}$ J. Temple, ${ }^{137}$ M. B. Tonjes,${ }^{137}$

S. C. Tonwar, ${ }^{137}$ A. Apyan, ${ }^{138}$ G. Bauer, ${ }^{138}$ W. Busza, ${ }^{138}$ E. Butz,${ }^{138}$ I. A. Cali, ${ }^{138}$ M. Chan, ${ }^{138}$ V. Dutta, ${ }^{138}$ G. Gomez Ceballos, ${ }^{138}$ M. Goncharov ${ }^{138}$ Y. Kim,,${ }^{138}$ M. Klute, ${ }^{138}$ A. Levin, ${ }^{138}$ P. D. Luckey, ${ }^{138}$ T. Ma,${ }^{138}$ S. Nahn, ${ }^{138}$ C. Paus, ${ }^{138}$ D. Ralph, ${ }^{138}$ C. Roland, ${ }^{138}$ G. Roland, ${ }^{138}$ G. S. F. Stephans, ${ }^{138}$ F. Stöckli, ${ }^{138}$ K. Sumorok, ${ }^{138}$ K. Sung, ${ }^{138}$ D. Velicanu, ${ }^{138}$ E. A. Wenger, ${ }^{138}$ R. Wolf, ${ }^{138}$ B. Wyslouch, ${ }^{138}$ M. Yang, ${ }^{138}$ Y. Yilmaz, ${ }^{138}$ A. S. Yoon, ${ }^{138}$ M. Zanetti, ${ }^{138}$ V. Zhukova, ${ }^{138}$ B. Dahmes, ${ }^{139}$ A. De Benedetti, ${ }^{139}$ G. Franzoni, ${ }^{139}$ A. Gude, ${ }^{139}$ S. C. Kao, ${ }^{139}$ K. Klapoetke, ${ }^{139}$

Y. Kubota, ${ }^{139}$ J. Mans,${ }^{139}$ N. Pastika, ${ }^{139}$ R. Rusack, ${ }^{139}$ M. Sasseville, ${ }^{139}$ A. Singovsky, ${ }^{139}$ N. Tambe, ${ }^{139}$

J. Turkewitz, ${ }^{139}$ L. M. Cremaldi, ${ }^{140}$ R. Kroeger, ${ }^{140}$ L. Perera, ${ }^{140}$ R. Rahmat,,${ }^{140}$ D. A. Sanders,,${ }^{140}$ E. Avdeeva, ${ }^{141}$

K. Bloom, ${ }^{141}$ S. Bose, ${ }^{141}$ D. R. Claes, ${ }^{141}$ A. Dominguez, ${ }^{141}$ M. Eads, ${ }^{141}$ J. Keller, ${ }^{141}$ I. Kravchenko, ${ }^{141}$

J. Lazo-Flores, ${ }^{141}$ S. Malik, ${ }^{141}$ G. R. Snow, ${ }^{141}$ A. Godshalk, ${ }^{142}$ I. Iashvilii, ${ }^{142}$ S. Jain, ${ }^{142}$ A. Kharchilava, ${ }^{142}$

A. Kumar, ${ }^{142}$ S. Rappoccio, ${ }^{142}$ Z. Wan, ${ }^{142}$ G. Alverson, ${ }^{143}$ E. Barberis, ${ }^{143}$ D. Baumgartel, ${ }^{143}$ M. Chasco, ${ }^{143}$ J. Haley, ${ }^{143}$ D. Nash, ${ }^{143}$ T. Orimoto, ${ }^{143}$ D. Trocino, ${ }^{143}$ D. Wood, ${ }^{143}$ J. Zhang, ${ }^{143}$ A. Anastassov,${ }^{144}$ K. A. Hahn, ${ }^{144}$ A. Kubik, ${ }^{144}$ L. Lusito, ${ }^{144}$ N. Mucia, ${ }^{144}$ N. Odell, ${ }^{144}$ R. A. Ofierzynski, ${ }^{144}$ B. Pollack, ${ }^{144}$ A. Pozdnyakov, ${ }^{144}$ M. Schmitt, ${ }^{144}$ S. Stoynev, ${ }^{144}$ M. Velasco, ${ }^{144}$ S. Won, ${ }^{144}$ D. Berry, ${ }^{145}$ A. Brinkerhoff, ${ }^{145}$ K. M. Chan, ${ }^{145}$ M. Hildreth, ${ }^{145}$ C. Jessop, ${ }^{145}$ D. J. Karmgard,${ }^{145}$ J. Kolb, ${ }^{145}$ K. Lannon, ${ }^{145}$ W. Luo, ${ }^{145}$ S. Lynch, ${ }^{145}$ N. Marinelli, ${ }^{145}$ D. M. Morse, ${ }^{145}$ T. Pearson, ${ }^{145}$ M. Planer, ${ }^{145}$ R. Ruchti, ${ }^{145}$ J. Slaunwhite, ${ }^{145}$ N. Valls, ${ }^{145}$ M. Wayne, ${ }^{145}$ M. Wolf, ${ }^{145}$ L. Antonelli, ${ }^{146}$ B. Bylsma, ${ }^{146}$ L. S. Durkin, ${ }^{146}$ C. Hill, ${ }^{146}$ R. Hughes,${ }^{146}$ K. Kotov, ${ }^{146}$ T. Y. Ling, ${ }^{146}$ D. Puigh, ${ }^{146}$ M. Rodenburg, ${ }^{146}$ G. Smith, ${ }^{146}$ C. Vuosalo, ${ }^{146}$ G. Williams, ${ }^{146}$ B. L. Winer, ${ }^{146}$ E. Berry, ${ }^{147}$ P. Elmer, ${ }^{147}$ V. Halyo, ${ }^{147}$ P. Hebda, ${ }^{147}$ J. Hegeman, ${ }^{147}$ A. Hunt, ${ }^{147}$ P. Jindal, ${ }^{147}$ S. A. Koay, ${ }^{147}$ D. Lopes Pegna ${ }^{147}$ P. Lujan, ${ }^{147}$ D. Marlow, ${ }^{147}$ T. Medvedeva, ${ }^{147}$ M. Mooney, ${ }^{147}$ J. Olsen, ${ }^{147}$ P. Piroué, ${ }^{147}$ X. Quan, ${ }^{147}$ A. Raval, ${ }^{147}$ H. Saka, ${ }^{147}$ D. Stickland,${ }^{147}$ C. Tully, ${ }^{147}$ J. S. Werner, ${ }^{147}$ S. C. Zenz,${ }^{147}$ A. Zuranski, ${ }^{147}$ E. Brownson, ${ }^{148}$ A. Lopez ${ }^{148}$ H. Mendez,${ }^{148}$ J.E. Ramirez Vargas, ${ }^{148}$ E. Alagoz, ${ }^{149}$ V.E. Barnes, ${ }^{149}$ D. Benedetti, ${ }^{149}$ G. Bolla, ${ }^{149}$ D. Bortoletto, ${ }^{149}$ 
M. De Mattia, ${ }^{149}$ A. Everett, ${ }^{149}$ Z. Hu, ${ }^{149}$ M. Jones, ${ }^{149}$ O. Koybasi,${ }^{149}$ M. Kress, ${ }^{149}$ A. T. Laasanen, ${ }^{149}$ N. Leonardo, ${ }^{149}$ V. Maroussov, ${ }^{149}$ P. Merkel, ${ }^{149}$ D. H. Miller ${ }^{149}$ N. Neumeister, ${ }^{149}$ I. Shipsey, ${ }^{149}$ D. Silvers, ${ }^{149}$ A. Svyatkovskiy, ${ }^{149}$ M. Vidal Marono, ${ }^{149}$ H. D. Yoo, ${ }^{149}$ J. Zablocki, ${ }^{149}$ Y. Zheng, ${ }^{149}$ S. Guragain, ${ }^{150}$ N. Parashar ${ }^{150}$ A. Adair, ${ }^{151}$ B. Akgun, ${ }^{151}$ C. Boulahouache, ${ }^{151}$ K. M. Ecklund, ${ }^{151}$ F. J. M. Geurts, ${ }^{151}$ W. Li, ${ }^{151}$ B. P. Padley, ${ }^{151}$ R. Redjimi, ${ }^{151}$ J. Roberts, ${ }^{151}$ J. Zabel, ${ }^{151}$ B. Betchart, ${ }^{152}$ A. Bodek, ${ }^{152}$ Y. S. Chung, ${ }^{152}$ R. Covarelli, ${ }^{152}$ P. de Barbaro, ${ }^{152}$ R. Demina, ${ }^{152}$ Y. Eshaq, ${ }^{152}$ T. Ferbel, ${ }^{152}$ A. Garcia-Bellido, ${ }^{152}$ P. Goldenzweig, ${ }^{152}$ J. Han, ${ }^{152}$ A. Harel, ${ }^{152}$ D. C. Miner, ${ }^{152}$ D. Vishnevskiy, ${ }^{152}$ M. Zielinski, ${ }^{152}$ A. Bhatti, ${ }^{153}$ R. Ciesielski, ${ }^{153}$ L. Demortier,${ }^{153}$ K. Goulianos, ${ }^{153}$ G. Lungu, ${ }^{153}$ S. Malik, ${ }^{153}$ C. Mesropian, ${ }^{153}$ S. Arora, ${ }^{154}$ A. Barker, ${ }^{154}$ J. P. Chou, ${ }^{154}$ C. Contreras-Campana, ${ }^{154}$ E. Contreras-Campana, ${ }^{154}$ D. Duggan, ${ }^{154}$ D. Ferencek, ${ }^{154}$ Y. Gershtein, ${ }^{154}$ R. Gray, ${ }^{154}$ E. Halkiadakis, ${ }^{154}$ D. Hidas, ${ }^{154}$ A. Lath,${ }^{154}$ S. Panwalkar, ${ }^{154}$ M. Park, ${ }^{154}$ R. Patel,${ }^{154}$ V. Rekovic, ${ }^{154}$ J. Robles, ${ }^{154}$ K. Rose, ${ }^{154}$ S. Salur, ${ }^{154}$ S. Schnetzer,${ }^{154}$ C. Seitz, ${ }^{154}$ S. Somalwar, ${ }^{154}$ R. Stone,${ }^{154}$ S. Thomas, ${ }^{154}$ M. Walker,${ }^{154}$ G. Cerizza, ${ }^{155}$ M. Hollingsworth, ${ }^{155}$ S. Spanier, ${ }^{155}$ Z. C. Yang, ${ }^{155}$ A. York, ${ }^{155}$ R. Eusebi, ${ }^{156}$ W. Flanagan, ${ }^{156}$ J. Gilmore, ${ }^{156}$ T. Kamon, ${ }^{156 \text {,hhh }}$ V. Khotilovich, ${ }^{156}$ R. Montalvo, ${ }^{156}$ I. Osipenkov, ${ }^{156}$ Y. Pakhotin, ${ }^{156}$ A. Perloff, ${ }^{156}$ J. Roe, ${ }^{156}$ A. Safonov, ${ }^{156}$ T. Sakuma, ${ }^{156}$ S. Sengupta, ${ }^{156}$ I. Suarez, ${ }^{156}$ A. Tatarinov, ${ }^{156}$ D. Toback, ${ }^{156}$ N. Akchurin,,${ }^{157}$ J. Damgov, ${ }^{157}$ C. Dragoiu, ${ }^{157}$ P. R. Dudero, ${ }^{157}$ C. Jeong, ${ }^{157}$ K. Kovitanggoon, ${ }^{157}$ S. W. Lee, ${ }^{157}$ T. Libeiro, ${ }^{157}$ I. Volobouev, ${ }^{157}$ E. Appelt, ${ }^{158}$ A. G. Delannoy, ${ }^{158}$ C. Florez, ${ }^{158}$ S. Greene, ${ }^{158}$ A. Gurrola, ${ }^{158}$ W. Johns, ${ }^{158}$ P. Kurt, ${ }^{158}$ C. Maguire, ${ }^{158}$ A. Melo, ${ }^{158}$ M. Sharma, ${ }^{158}$ P. Sheldon, ${ }^{158}$ B. Snook,${ }^{158}$ S. Tuo, ${ }^{158}$ J. Velkovska, ${ }^{158}$ M. W. Arenton, ${ }^{159}$ M. Balazs, ${ }^{159}$ S. Boutle ${ }^{159}$ B. Cox, ${ }^{159}$ B. Francis, ${ }^{159}$ J. Goodell, ${ }^{159}$ R. Hirosky, ${ }^{159}$ A. Ledovskoy, ${ }^{159}$ C. Lin, ${ }^{159}$

C. Neu, ${ }^{159}$ J. Wood, ${ }^{159}$ S. Gollapinni, ${ }^{160}$ R. Harr, ${ }^{160}$ P. E. Karchin, ${ }^{160}$ C. Kottachchi Kankanamge Don, ${ }^{160}$ P. Lamichhane, ${ }^{160}$ A. Sakharov,${ }^{160}$ M. Anderson, ${ }^{161}$ D. A. Belknap, ${ }^{161}$ L. Borrello, ${ }^{161}$ D. Carlsmith, ${ }^{161}$ M. Cepeda, ${ }^{161}$ S. Dasu, ${ }^{161}$ E. Friis, ${ }^{161}$ L. Gray, ${ }^{161}$ K. S. Grogg, ${ }^{161}$ M. Grothe, ${ }^{161}$ R. Hall-Wilton, ${ }^{161}$ M. Herndon, ${ }^{161}$ A. Hervé, ${ }^{161}$ P. Klabbers, ${ }^{161}$ J. Klukas, ${ }^{161}$ A. Lanaro, ${ }^{161}$ C. Lazaridis,${ }^{161}$ R. Loveless, ${ }^{161}$ A. Mohapatra, ${ }^{161}$ M. U. Mozer, ${ }^{161}$ I. Ojalvo, ${ }^{161}$ F. Palmonari, ${ }^{161}$ G. A. Pierro, ${ }^{161}$ I. Ross, ${ }^{161}$ A. Savin, ${ }^{161}$ W. H. Smith, ${ }^{161}$ and J. Swanson ${ }^{161}$

(CMS Collaboration)

\author{
${ }^{1}$ Yerevan Physics Institute, Yerevan, Armenia \\ ${ }^{2}$ Institut für Hochenergiephysik der OeAW, Wien, Austria \\ ${ }^{3}$ National Centre for Particle and High Energy Physics, Minsk, Belarus \\ ${ }^{4}$ Universiteit Antwerpen, Antwerpen, Belgium \\ ${ }^{5}$ Vrije Universiteit Brussel, Brussel, Belgium \\ ${ }^{6}$ Université Libre de Bruxelles, Bruxelles, Belgium \\ ${ }^{7}$ Ghent University, Ghent, Belgium \\ ${ }^{8}$ Université Catholique de Louvain, Louvain-la-Neuve, Belgium \\ ${ }^{9}$ Université de Mons, Mons, Belgium \\ ${ }^{10}$ Centro Brasileiro de Pesquisas Fisicas, Rio de Janeiro, Brazil \\ ${ }^{11}$ Universidade do Estado do Rio de Janeiro, Rio de Janeiro, Brazil \\ ${ }^{12 a}$ Universidade Estadual Paulista, São Paulo, Brazil \\ ${ }^{12 \mathrm{~b}}$ Universidade Federal do ABC, São Paulo, Brazil \\ ${ }^{13}$ Institute for Nuclear Research and Nuclear Energy, Sofia, Bulgaria \\ ${ }^{14}$ University of Sofia, Sofia, Bulgaria \\ ${ }^{15}$ Institute of High Energy Physics, Beijing, China \\ ${ }^{16}$ State Key Laboratory of Nuclear Physics and Technology, Peking University, Beijing, China \\ ${ }^{17}$ Universidad de Los Andes, Bogota, Colombia \\ ${ }^{18}$ Technical University of Split, Split, Croatia \\ ${ }^{19}$ University of Split, Split, Croatia \\ ${ }^{20}$ Institute Rudjer Boskovic, Zagreb, Croatia \\ ${ }^{21}$ University of Cyprus, Nicosia, Cyprus \\ ${ }^{22}$ Charles University, Prague, Czech Republic \\ ${ }^{23}$ Academy of Scientific Research and Technology of the Arab Republic of Egypt, \\ Egyptian Network of High Energy Physics, Cairo, Egypt \\ ${ }^{24}$ National Institute of Chemical Physics and Biophysics, Tallinn, Estonia \\ ${ }^{25}$ Department of Physics, University of Helsinki, Helsinki, Finland \\ ${ }^{26}$ Helsinki Institute of Physics, Helsinki, Finland \\ ${ }^{27}$ Lappeenranta University of Technology, Lappeenranta, Finland \\ ${ }^{28}$ DSM/IRFU, CEA/Saclay, Gif-sur-Yvette, France
}


${ }^{29}$ Laboratoire Leprince-Ringuet, Ecole Polytechnique, IN2P3-CNRS, Palaiseau, France

${ }^{30}$ Institut Pluridisciplinaire Hubert Curien, Université de Strasbourg,

Université de Haute Alsace Mulhouse, CNRS/IN2P3, Strasbourg, France

${ }^{31}$ Université de Lyon, Université Claude Bernard Lyon 1, CNRS-IN2P3, Institut de Physique Nucléaire de Lyon, Villeurbanne, France

${ }^{32}$ Institute of High Energy Physics and Informatization, Tbilisi State University, Tbilisi, Georgia

${ }^{33}$ RWTH Aachen University, I. Physikalisches Institut, Aachen, Germany

${ }^{34}$ RWTH Aachen University, III. Physikalisches Institut A, Aachen, Germany

${ }^{35}$ RWTH Aachen University, III. Physikalisches Institut B, Aachen, Germany

${ }^{36}$ Deutsches Elektronen-Synchrotron, Hamburg, Germany

${ }^{37}$ University of Hamburg, Hamburg, Germany

${ }^{38}$ Institut für Experimentelle Kernphysik, Karlsruhe, Germany

${ }^{39}$ Institute of Nuclear Physics “Demokritos,” Aghia Paraskevi, Greece

${ }^{40}$ University of Athens, Athens, Greece

${ }^{41}$ University of Ioánnina, Ioánnina, Greece

${ }^{42}$ KFKI Research Institute for Particle and Nuclear Physics, Budapest, Hungary

${ }^{43}$ Institute of Nuclear Research ATOMKI, Debrecen, Hungary

${ }^{44}$ University of Debrecen, Debrecen, Hungary

${ }^{45}$ Panjab University, Chandigarh, India

${ }^{46}$ University of Delhi, Delhi, India

${ }^{47}$ Saha Institute of Nuclear Physics, Kolkata, India

${ }^{48}$ Bhabha Atomic Research Centre, Mumbai, India

${ }^{49}$ Tata Institute of Fundamental Research-EHEP, Mumbai, India

${ }^{50}$ Tata Institute of Fundamental Research-HECR, Mumbai, India

${ }^{51}$ Institute for Research in Fundamental Sciences (IPM), Tehran, Iran

${ }^{52 \mathrm{a}}$ INFN Sezione di Bari, Bari, Italy

${ }^{52 \mathrm{~b}}$ Università di Bari, Bari, Italy

${ }^{52 \mathrm{c}}$ Politecnico di Bari, Bari, Italy

${ }^{53}$ INFN Sezione di Bologna, Bologna, Italy

${ }^{53 \mathrm{~b}}$ Università di Bologna, Bologna, Italy

${ }^{54 a}$ INFN Sezione di Catania, Catania, Italy

${ }^{54 \mathrm{~b}}$ Università di Catania, Catania, Italy

${ }^{55}$ a INFN Sezione di Firenze, Firenze, Italy

${ }^{55 \mathrm{~b}}$ Università di Firenze, Firenze, Italy

${ }^{56}$ INFN Laboratori Nazionali di Frascati, Frascati, Italy

${ }^{57 a}$ INFN Sezione di Genova, Genova, Italy

${ }^{57 b}$ Università di Genova, Genova, Italy

${ }^{58}$ a INFN Sezione di Milano-Bicocca, Milano, Italy

${ }^{58 \mathrm{~b}}$ Università di Milano-Bicocca, Milano, Italy

${ }^{59 a}$ INFN Sezione di Napoli, Napoli, Italy

${ }^{59 b}$ Università di Napoli "Federico II,", Napoli, Italy

${ }^{59 c}$ Università della Basilicata (Potenza), Napoli, Italy

${ }^{59 \mathrm{~d}}$ Università G. Marconi (Roma), Napoli, Italy

${ }^{60 a}$ INFN Sezione di Padova, Padova, Italy

${ }^{60 \mathrm{~b}}$ Università di Padova, Padova, Italy

${ }^{60 \mathrm{c}}$ Università di Trento (Trento), Padova, Italy

${ }^{61 a}$ INFN Sezione di Pavia, Pavia, Italy

${ }^{61 \mathrm{~b}}$ Università di Pavia, Pavia, Italy

${ }^{62 \mathrm{a}}$ INFN Sezione di Perugia, Perugia, Italy

${ }^{62 \mathrm{~b}}$ Università di Perugia, Perugia, Italy

${ }^{63 a}$ INFN Sezione di Pisa, Pisa, Italy

${ }^{63 \mathrm{~b}}$ Università di Pisa, Pisa, Italy

${ }^{63 \mathrm{c}}$ Scuola Normale Superiore di Pisa, Pisa, Italy

${ }^{64 a}$ INFN Sezione di Roma, Roma, Italy

${ }^{64 \mathrm{~b}}$ Università di Roma, Roma, Italy

${ }^{65 a}$ INFN Sezione di Torino, Torino, Italy

${ }^{65 \mathrm{~b}}$ Università di Torino, Torino, Italy

${ }^{65 \mathrm{c}}$ Università del Piemonte Orientale (Novara), Torino, Italy

${ }^{66 \mathrm{a}}$ INFN Sezione di Trieste, Trieste, Italy

${ }^{66 \mathrm{~b}}$ Università di Trieste, Trieste, Italy

${ }^{67}$ Kangwon National University, Chunchon, Korea

${ }^{68}$ Kyungpook National University, Daegu, Korea 
${ }^{69}$ Chonnam National University, Institute for Universe and Elementary Particles, Kwangju, Korea

${ }^{70}$ Korea University, Seoul, Korea

${ }^{71}$ University of Seoul, Seoul, Korea

${ }^{72}$ Sungkyunkwan University, Suwon, Korea

${ }^{73}$ Vilnius University, Vilnius, Lithuania

${ }^{74}$ Centro de Investigacion y de Estudios Avanzados del IPN, Mexico City, Mexico

${ }^{75}$ Universidad Iberoamericana, Mexico City, Mexico

${ }^{76}$ Benemerita Universidad Autonoma de Puebla, Puebla, Mexico

${ }^{77}$ Universidad Autónoma de San Luis Potosí, San Luis Potosí, Mexico

${ }^{78}$ University of Auckland, Auckland, New Zealand

${ }^{79}$ University of Canterbury, Christchurch, New Zealand

${ }^{80}$ National Centre for Physics, Quaid-I-Azam University, Islamabad, Pakistan

${ }^{81}$ National Centre for Nuclear Research, Swierk, Poland

${ }^{82}$ Institute of Experimental Physics, Faculty of Physics, University of Warsaw, Warsaw, Poland

${ }^{83}$ Laboratório de Instrumentação e Física Experimental de Partículas, Lisboa, Portugal

${ }^{84}$ Joint Institute for Nuclear Research, Dubna, Russia

${ }^{85}$ Petersburg Nuclear Physics Institute, Gatchina (St. Petersburg), Russia

${ }^{86}$ Institute for Nuclear Research, Moscow, Russia

${ }^{87}$ Institute for Theoretical and Experimental Physics, Moscow, Russia

${ }^{88}$ Moscow State University, Moscow, Russia

${ }^{89}$ P. N. Lebedev Physical Institute, Moscow, Russia

${ }^{90}$ State Research Center of Russian Federation, Institute for High Energy Physics, Protvino, Russia

${ }^{91}$ University of Belgrade, Faculty of Physics and Vinca Institute of Nuclear Sciences, Belgrade, Serbia

${ }^{92}$ Centro de Investigaciones Energéticas Medioambientales y Tecnológicas (CIEMAT), Madrid, Spain

${ }^{93}$ Universidad Autónoma de Madrid, Madrid, Spain

${ }^{94}$ Universidad de Oviedo, Oviedo, Spain

${ }^{95}$ Instituto de Física de Cantabria (IFCA), CSIC-Universidad de Cantabria, Santander, Spain

${ }^{96}$ CERN, European Organization for Nuclear Research, Geneva, Switzerland

${ }^{97}$ Paul Scherrer Institut, Villigen, Switzerland

${ }^{98}$ Institute for Particle Physics, ETH Zurich, Zurich, Switzerland

${ }^{99}$ Universität Zürich, Zurich, Switzerland

${ }^{100}$ National Central University, Chung-Li, Taiwan

${ }^{101}$ National Taiwan University (NTU), Taipei, Taiwan

${ }^{102}$ Chulalongkorn University, Bangkok, Thailand

${ }^{103}$ Cukurova University, Adana, Turkey

${ }^{104}$ Middle East Technical University, Physics Department, Ankara, Turkey

${ }^{105}$ Bogazici University, Istanbul, Turkey

${ }^{106}$ Istanbul Technical University, Istanbul, Turkey

${ }^{107}$ National Scientific Center, Kharkov Institute of Physics and Technology, Kharkov, Ukraine

${ }^{108}$ University of Bristol, Bristol, United Kingdom

${ }^{109}$ Rutherford Appleton Laboratory, Didcot, United Kingdom

${ }^{110}$ Imperial College, London, United Kingdom

${ }^{111}$ Brunel University, Uxbridge, United Kingdom

${ }^{112}$ Baylor University, Waco, Texas 76706, USA

${ }^{113}$ The University of Alabama, Tuscaloosa, Alabama 35487, USA

${ }^{114}$ Boston University, Boston, Massachusetts 02215, USA

${ }^{115}$ Brown University, Providence, Rhode Island 02912, USA

${ }^{116}$ University of California, Davis, Davis, California 95616, USA

${ }^{117}$ University of California, Los Angeles, California 90095, USA

${ }^{118}$ University of California, Riverside, Riverside, California 92521, USA

${ }^{119}$ University of California, San Diego, La Jolla, California 92093, USA

${ }^{120}$ University of California, Santa Barbara, Santa Barbara, California 93106, USA

${ }^{121}$ California Institute of Technology, Pasadena, California 91125, USA

${ }^{122}$ Carnegie Mellon University, Pittsburgh, Pennsylvania 15213, USA

${ }^{123}$ University of Colorado at Boulder, Boulder, Colorado 80309, USA

${ }^{124}$ Cornell University, Ithaca, New York 14853, USA

${ }^{125}$ Fairfield University, Fairfield, Connecticut 06824, USA

${ }^{126}$ Fermi National Accelerator Laboratory, Batavia, Illinois 6051, USA

${ }^{127}$ University of Florida, Gainesville, Florida 3261, USA

${ }^{128}$ Florida International University, Miami, Florida 33199, USA

${ }^{129}$ Florida State University, Tallahassee, Florida 32306, USA 
${ }^{130}$ Florida Institute of Technology, Melbourne, Florida 32901, USA

${ }^{131}$ University of Illinois at Chicago (UIC), Chicago, Illinois 60607, USA

${ }^{132}$ The University of Iowa, Iowa City, Iowa 52242, USA

${ }^{133}$ Johns Hopkins University, Baltimore, Maryland 21218, USA

${ }^{134}$ The University of Kansas, Lawrence, Kansas 66045, USA

${ }^{135}$ Kansas State University, Manhattan, Kansas 66506, USA

${ }^{136}$ Lawrence Livermore National Laboratory, Livermore, California 94720, USA

${ }^{137}$ University of Maryland, College Park, Maryland 20742, USA

${ }^{138}$ Massachusetts Institute of Technology, Cambridge, Massachusetts 02139, USA

${ }^{139}$ University of Minnesota, Minneapolis, Minnesota 55455, USA

${ }^{140}$ University of Mississippi, Oxford, Mississippi 38677, USA

${ }^{141}$ University of Nebraska-Lincoln, Lincoln, Nebraska 68588, USA

${ }^{142}$ State University of New York at Buffalo, Buffalo, New York 14260, USA

${ }^{143}$ Northeastern University, Boston, Massachusetts 02115, USA

${ }^{144}$ Northwestern University, Evanston, Illinois 60208, USA

${ }^{145}$ University of Notre Dame, Notre Dame, Indiana 46556, USA

${ }^{146}$ The Ohio State University, Columbus, Ohio 43210, USA

${ }^{147}$ Princeton University, Princeton, New Jersey 08544, USA

${ }^{148}$ University of Puerto Rico, Mayaguez, Puerto Rico 00680

${ }^{149}$ Purdue University, West Lafayette, Indiana 47907, USA

${ }^{150}$ Purdue University Calumet, Hammond, Indiana 46323, USA

${ }^{151}$ Rice University, Houston, Texas 77251, USA

${ }^{152}$ University of Rochester, Rochester, New York 14627, USA

${ }^{153}$ The Rockefeller University, New York, New York 10021, USA

${ }^{154}$ Rutgers, the State University of New Jersey, Piscataway, New Jersey 08854, USA

${ }^{155}$ University of Tennessee, Knoxville, Tennessee 37996, USA

${ }^{156}$ Texas A\&M University, College Station, Texas 77843, USA

${ }^{157}$ Texas Tech University, Lubbock, Texas 79409, USA

${ }^{158}$ Vanderbilt University, Nashville, Tennessee 37235, USA

${ }^{159}$ University of Virginia, Charlottesville, Virginia 22901, USA

${ }^{160}$ Wayne State University, Detroit, Michigan 48202, USA

${ }^{161}$ University of Wisconsin, Madison, Wisconsin 53706, USA

${ }^{\mathrm{a}}$ Deceased.

${ }^{\mathrm{b}}$ Also at Vienna University of Technology, Vienna, Austria.

${ }^{\mathrm{c}}$ Also at CERN, European Organization for Nuclear Research, Geneva, Switzerland.

${ }^{\mathrm{d}}$ Also at National Institute of Chemical Physics and Biophysics, Tallinn, Estonia.

eAlso at Universidade Estadual de Campinas, Campinas, Brazil.

${ }_{\mathrm{f}}^{\mathrm{f}}$ Also at California Institute of Technology, Pasadena, California, USA.

${ }^{\mathrm{g}}$ Also at Laboratoire Leprince-Ringuet, Ecole Polytechnique, IN2P3-CNRS, Palaiseau, France.

${ }^{\mathrm{h}}$ Also at Suez Canal University, Suez, Egypt.

${ }^{\mathrm{i}}$ Also at Zewail City of Science and Technology, Zewail, Egypt.

${ }^{\mathrm{j} A l s o}$ at Cairo University, Cairo, Egypt.

${ }^{\mathrm{k}}$ Also at Fayoum University, El-Fayoum, Egypt.

${ }^{1}$ Also at British University in Egypt, Cairo, Egypt.

${ }^{\mathrm{m}}$ Present address: Ain Shams University, Cairo, Egypt.

${ }^{\mathrm{n}}$ Also at National Centre for Nuclear Research, Swierk, Poland.

${ }^{\circ}$ Also at Université de Haute-Alsace, Mulhouse, France.

${ }^{\mathrm{p}}$ Also at Joint Institute for Nuclear Research, Dubna, Russia.

${ }^{\mathrm{q}}$ Also at Moscow State University, Moscow, Russia.

${ }^{\mathrm{r}}$ Also at Brandenburg University of Technology, Cottbus, Germany.

${ }^{\mathrm{s}}$ Also at The University of Kansas, Lawrence, Kansas, USA.

${ }^{\mathrm{t}}$ Also at Institute of Nuclear Research ATOMKI, Debrecen, Hungary.

${ }^{u}$ Also at Eötvös Loránd University, Budapest, Hungary.

${ }^{\mathrm{v}}$ Also at Tata Institute of Fundamental Research-HECR, Mumbai, India.

${ }^{\text {w}}$ Present address: King Abdulaziz University, Jeddah, Saudi Arabia.

${ }^{\mathrm{x}}$ Also at University of Visva-Bharati, Santiniketan, India.

${ }^{\mathrm{y}}$ Also at Sharif University of Technology, Tehran, Iran. 
${ }^{\mathrm{z}}$ Also at Isfahan University of Technology, Isfahan, Iran.

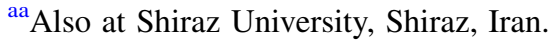

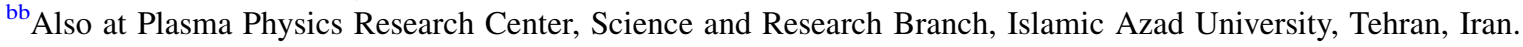

${ }^{\mathrm{cc}}$ Also at Facoltà Ingegneria, Università di Roma, Roma, Italy.

${ }^{\mathrm{dd}}$ Also at Università degli Studi di Siena, Siena, Italy.

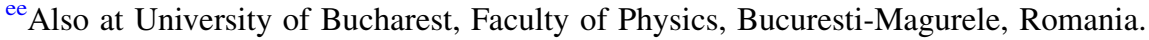

${ }^{\mathrm{ff}}$ Also at Faculty of Physics of University of Belgrade, Belgrade, Serbia.

${ }^{\mathrm{gg}}$ Also at University of California, Los Angeles, California, USA.

${ }^{\text {hh }}$ Also at Scuola Normale e Sezione dell'INFN, Pisa, Italy.

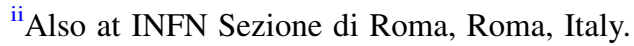

${ }^{\mathrm{jj}}$ Also at University of Athens, Athens, Greece.

${ }^{\mathrm{kk}}$ Also at Rutherford Appleton Laboratory, Didcot, United Kingdom.

${ }^{11}$ Also at Paul Scherrer Institut, Villigen, Switzerland.

${ }^{\mathrm{mm}}$ Also at Institute for Theoretical and Experimental Physics, Moscow, Russia.

${ }^{n n}$ Also at Albert Einstein Center for Fundamental Physics, Bern, Switzerland.

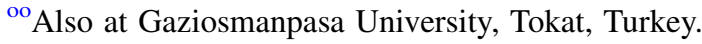

${ }^{\mathrm{pp}}$ Also at Adiyaman University, Adiyaman, Turkey.

${ }^{\mathrm{qq}}$ Also at Izmir Institute of Technology, Izmir, Turkey.

${ }^{\mathrm{rr}}$ Also at The University of Iowa, Iowa City, Iowa, USA.

${ }^{\mathrm{ss}}$ Also at Mersin University, Mersin, Turkey.

${ }^{\text {tt} A l s o ~ a t ~ O z y e g i n ~ U n i v e r s i t y, ~ I s t a n b u l, ~ T u r k e y . ~}$

${ }^{\mathrm{uu}}$ Also at Kafkas University, Kars, Turkey.

${ }^{\mathrm{vv}}$ Also at Suleyman Demirel University, Isparta, Turkey.

${ }^{\text {ww }}$ Also at Ege University, Izmir, Turkey.

${ }^{\mathrm{xx}}$ Also at Mimar Sinan University, Istanbul, Istanbul, Turkey.

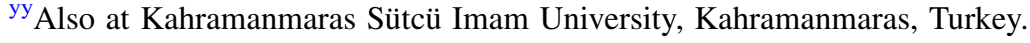

${ }^{z z}$ Also at School of Physics and Astronomy, University of Southampton, Southampton, United Kingdom.

${ }^{\text {aaa } A l s o ~ a t ~ I N F N ~ S e z i o n e ~ d i ~ P e r u g i a, ~ U n i v e r s i t a ̀ ~ d i ~ P e r u g i a, ~ P e r u g i a, ~ I t a l y . ~}$

${ }^{\mathrm{bbb}}$ Also at Utah Valley University, Orem, Utah, USA.

${ }^{\mathrm{cc}}$ Present address: University of Edinburgh, Scotland, Edinburgh, United Kingdom.

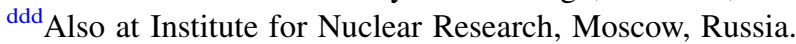

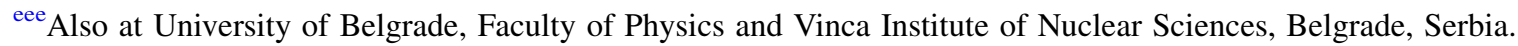

${ }^{\mathrm{fff}}$ Also at Argonne National Laboratory, Argonne, Illinois, USA.

${ }^{\text {ggg }}$ Also at Erzincan University, Erzincan, Turkey.

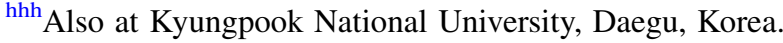

